\title{
Experimental investigation of cylindrical converging shock waves interacting with a polygonal heavy gas cylinder
}

\author{
Ting $\mathrm{Si}^{1}$, Tong Long ${ }^{1}$, Zhigang Zhai ${ }^{1}$ and Xisheng Luo ${ }^{1,} \dagger$ \\ ${ }^{1}$ Advanced Propulsion Laboratory, Department of Modern Mechanics, University of Science and \\ Technology of China, Hefei 230026, China
}

(Received 21 September 2014; revised 21 September 2015; accepted 1 October 2015; first published online 4 November 2015)

The interaction of cylindrical converging shock waves with a polygonal heavy gas cylinder is studied experimentally in a vertical annular diaphragmless shock tube. The reliability of the shock tube facility is verified in advance by capturing the cylindrical shock movements during the convergence and reflection processes using high-speed schlieren photography. Three types of air/ $\mathrm{SF}_{6}$ polygonal interfaces with cross-sections of an octagon, a square and an equilateral triangle are formed by the soap film technique. A high-speed laser sheet imaging method is employed to monitor the evolution of the three polygonal interfaces subjected to the converging shock waves. In the experiments, the Mach number of the incident cylindrical shock at its first contact with each interface is maintained to be 1.35 for all three cases. The results show that the evolution of the polygonal interfaces is heavily dependent on the initial conditions, such as the interface shapes and the shock features. A theoretical model for circulation initially deposited along the air/ $\mathrm{SF}_{6}$ polygonal interface is developed based on the theory of Samtaney \& Zabusky (J. Fluid Mech., vol. 269, 1994, pp. 45-78). The circulation depositions along the initial interface result in the differences in flow features among the three polygonal interfaces, including the interface velocities and the perturbation growth rates. In comparison with planar shock cases, there are distinct phenomena caused by the convergence effects, including the variation of shock strength during imploding and exploding (geometric convergence), consecutive reshocks on the interface (compressibility), and special behaviours of the movement of the interface structures (phase inversion).

Key words: compressible flows, shock waves

\section{Introduction}

Richtmyer-Meshkov (RM) instability occurs when an initially perturbed interface separating two different fluids is impulsively accelerated by a shock wave. The pioneering analysis on this shock-induced interfacial instability was performed 
theoretically and numerically by Richtmyer (1960) and then confirmed experimentally by Meshkov (1969). From then on, the RM instability has been widely studied due to its extensive applications in scientific and industrial fields such as inertial confinement fusion (ICF) (Lindl, McCrory \& Campbell 1992), supernova explosion (Arnett et al. 1989) and mixing of fuels in the scramjet (Yang, Kubota \& Zukoski 1993). In the process of the RM instability, baroclinic vorticity will be generated in the flow field because of the misalignment between the density and pressure gradients, resulting in interpenetration and mixing between two fluids. In laboratory conditions, a planar shock wave can be accurately formed in an ordinary shock tube, while a converging shock wave, especially with a controllable shape, is difficult to obtain because of its non-uniformity and variability in shape and strength. Previous experimental works on the RM instability mainly focused on the planar shock case (Brouillette 2002; Ranjan, Oakley \& Bonazza 2011), with only a few exceptions (Hosseini \& Takayama 2005; Luo et al. 2014a; Si, Zhai \& Luo 2014a; Si et al. 2014b), although a great deal of effort has been taken to generate converging shock waves (Perry \& Kantrowitz 1951; Takayama, Kleine \& Gronig 1987; Apazidis \& Lesser 1996; Hosseini, Onodera \& Takayama 2000; Dimotakis \& Samtaney 2006; Zhai et al. 2010). It is known that the shock waves often maintain curved shapes in various applications (e.g. the ICF experiments), in which the converging shock wave is desirable for providing extremely high pressure and density near the focal point. Therefore, it is of great significance to study the interaction of a converging shock wave with a controllable interface in order to better understand the converging RM instability.

In recent decades, the generation of converging shock waves was mainly performed in shock tube environments. Cylindrical converging shock waves were generated for the first time by Perry \& Kantrowitz (1951) using a horizontal annular shock tube, which consists of a cone-shaped inner core and an outer cylindrical shell. Later, a vertical annular coaxial shock tube was constructed by the group of Professor Takayama (Takayama et al. 1987; Watanabe, Onodera \& Takayama 1995; Hosseini et al. 2000) and gradually modified to observe uniformly shaped toroidal shock waves (Hosseini \& Takayama 2005, 2010). A different shock tube with a conically shaped test section was built to study converging shock waves by the group of Professor Apazidis (Apazidis \& Lesser 1996; Apazidis et al. 2002; Kjellander, Tillmark \& Apazidis 2011). Moreover, Dimotakis \& Samtaney (2006) reported a gas lens technique that generated a converging shock wave in a two-dimensional wedge geometry and the characteristic of the shock tube was further generalized (Vandenboomgaerde \& Aymard 2011). Zhai et al. (2010, 2012) proposed an efficient method to transform a planar shock wave into a cylindrical one, which employed the shock dynamics theory to design a curved wall profile of the test section in an ordinary shock tube. Recently, RM instability experiments in this shock tube have also been performed to reveal the great potential of this experimental facility (Luo et al. 2014a; Si et al. 2014a,b).

Generating an initially perturbed interface is crucial for the experimental study of the converging shock-induced RM instability in the above-mentioned shock tubes. In the case of a planar shock, different techniques developed to form the initial interfaces have been reported in the literature. Typically, the interfaces of different shapes between two gases include spherical bubbles formed by a soap film (Haas \& Sturtevant 1987; Layes, Jourdan \& Houas 2003; Ranjan et al. 2005; Zhai et al. 2011; Si et al. 2012), circular gas cylinders (Haas \& Sturtevant 1987; Hosseini \& Takayama 2005; Tomkins et al. 2008; Luo et al. 2014b), elliptical gas cylinders 
(Zou et al. 2010), single-/multi-mode interfaces generated by vibration (Jacobs \& Sheeley 1995; Mariani et al. 2008; Long et al. 2009) or by a gas curtain (Balakumar et al. 2008; Orlicz et al. 2009; Balasubramanian et al. 2012), rectangular blocks generated by a microfilm membrane supported by a fine wire mesh (Bates, Nikiforakis \& Holder 2007), and three-dimensional interfaces with a minimum surface feature formed by the soap film technique free of supporting meshes (Luo, Wang \& Si 2013). Recently, some polygonal interfaces have been formed in our group using thin pins to restrict the soap film and the results indicate that the soap film is a flimsy but durable material during the interface formation (Wang, Si \& Luo 2013; Zhai et al. 2014; Luo et al. 2015).

In this work, we aim to study the interaction of different polygonal interfaces accelerated by cylindrical converging shock waves. A vertical annular diaphragmless shock tube (Hosseini et al. 2000; Hosseini \& Takayama 2005) is constructed and polygonal interfaces of different shapes including an octagon, a square and an equilateral triangle are employed. We mainly limit our attention to the convergence effects and the baroclinic mechanism of the cylindrically converging RM instability with large initial deformation of the gas interfaces. It should be mentioned that the turbulent mixing is very interesting and important in the study of RM instability (Dimotakis 2005; Tomkins et al. 2008; Balakumar et al. 2012; Lombardini, Pullin \& Meiron $2014 a, b$ ) but is outside the scope of the present work. Under the current conditions, the characteristics of the cylindrical converging shock are described first, and rich phenomena of the polygonal interfaces accelerated by the cylindrical converging shock and the reflected shock from the focal point, during the early stage of the RM instability, are obtained for the first time. The convergence effects on the RM instability in these experiments are emphasized, including the variation of shock strength in the processes of shock convergence and reflection, consecutive reshocks on the interface (reverberating rarefaction waves are usually observed in planar geometry), and special behaviours of the movement of the interface structures. Moreover, the circulation initially deposited each polygonal interface subjected to a cylindrical converging shock wave is calculated analytically and compared with the experimental observations.

\section{Experimental methods}

\subsection{Generation of cylindrical converging shock waves}

A vertical annular diaphragmless shock tube is constructed in our group based on the idea of Hosseini \& Takayama (2005). Figure 1 presents the overall description of the facility, which mainly consists of a bottom auxiliary high-pressure chamber, an outer high-pressure chamber (i.e. the driver section), an inner low-pressure chamber (i.e. the driven section) and a top test section. The driver and driven sections are made up of three coaxial tubes which are rigidly fixed on a very heavy base containing channels for pressurization and evacuation of auxiliary high-pressure gas. A ring-shaped rubber membrane is mounted between two bulging metal grid flanges connecting the driver and driven sections. The test section, with an inner height of $5 \mathrm{~mm}$, is located on the top of the driven section. The height of the driver section is $600 \mathrm{~mm}$ with an inner diameter of $180 \mathrm{~mm}$ and an outer diameter of $260 \mathrm{~mm}$. The driven section has a height of $1500 \mathrm{~mm}$ with an inner diameter of $140 \mathrm{~mm}$ and an outer diameter of $160 \mathrm{~mm}$. Prior to each experimental run, both the driver section and the auxiliary high-pressure chamber are charged by a high-pressure gas (i.e. air in this work). The pressure in the auxiliary high-pressure chamber is always higher than that in the driver 


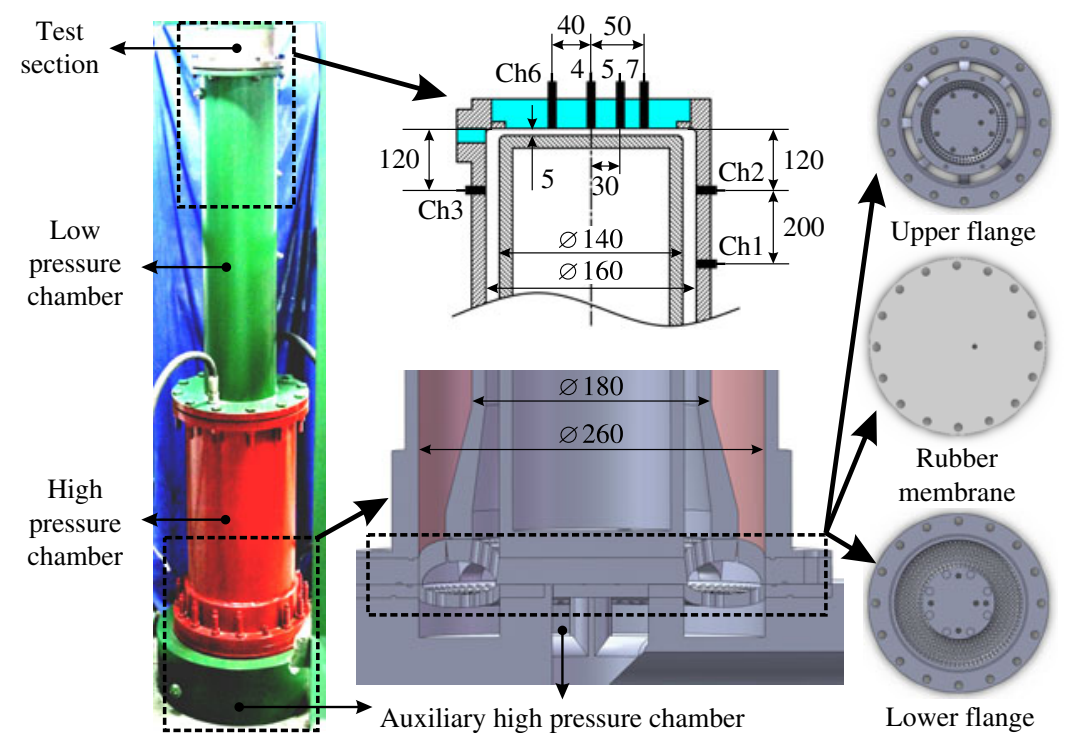

Figure 1. (Colour online) Photograph of the vertical annular diaphragmless shock tube and its detailed structures (unit: $\mathrm{mm}$ ), designed following a principle similar to that presented in Hosseini et al. (2000).

section to make sure that the rubber membrane is pressed onto the upper grid flange. Therefore, the driven section at atmospheric pressure is isolated from the driver section at high pressure. When diaphragm bursting happens at the exit of the auxiliary highpressure chamber to give a rapid pressure reduction, the rubber membrane suddenly moves to the lower grid flange and thus the high-pressure gas in the driver section exhausts to the driven section. In this way, an annular shock wave propagates in the driven section and a cylindrical shock wave is finally formed in the test section. To monitor the pressure histories in the annular driven section and the test section, several piezoelectric pressure transducers (QSY8117, Qishiyuan SCI. TECH. Co., Ltd, China, with measuring range $0-2 \mathrm{MPa}$, sensitivity $63 \mathrm{pC} / \mathrm{MPa}$, and rise time smaller than $2 \mu \mathrm{s}$ ) are used, and their seven locations (i.e. Ch1-Ch7) are also indicated in figure 1.

\subsection{Formation of initial polygonal interfaces}

In this work, we focus on the interface deformation and perturbation growth under cylindrical shock conditions by introducing large initial perturbations to a gas cylinder. For this purpose, three polygonal gas cylinders with different cross-sections, including an octagon, a square and an equilateral triangle, are formed by the soap film method (Wang et al. 2013). As shown in figure 2, very thin pins with diameter of $100 \mu \mathrm{m}$ are used as edges to connect the adjacent sides of the soap film to form polygonal gas cylinders in the test section. Generally, only one pin is needed at an edge with a sharp corner between two adjacent soap films when the corner angle is larger than $120^{\circ}$, while three pins should be employed for an edge with a corner angle smaller than $60^{\circ}$, and two pins for an edge with an intermediate corner angle $\left(60^{\circ}-120^{\circ}\right)$ (Isenberg 1992). Therefore, two pins are used at the edges with a sharp corner for the equilateral triangle and the square interfaces, and only one pin is used between two adjacent soap films for the octagon interface. The chamfers between the axis lines of two combined 
(a)

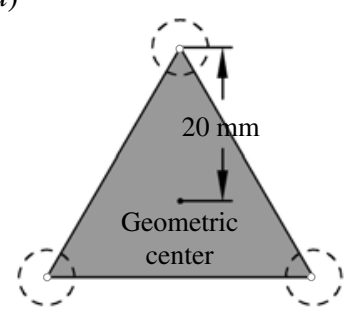

$(d)$

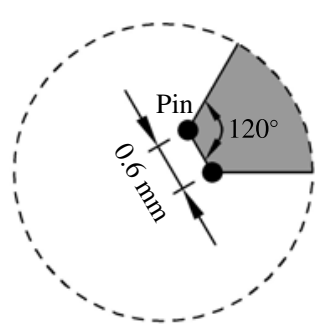

(b)

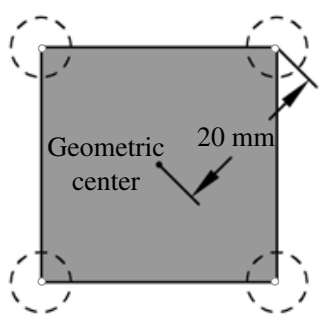

(e)

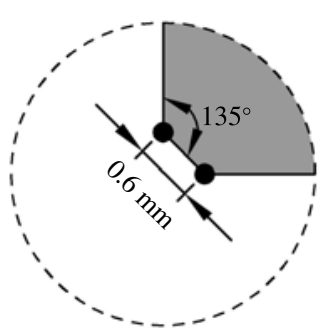

(c)

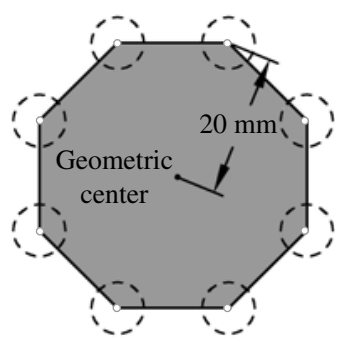

$(f)$

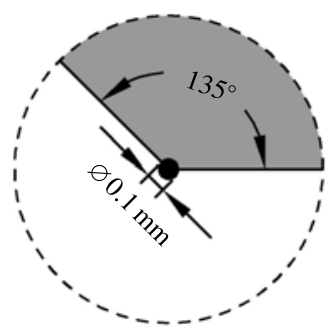

FIGURE 2. Three different gas cylinder configurations $(a-c)$ and corresponding corner details $(d-f)$, with cross-sections of an equilateral triangle $(a, d)$, a square $(b, e)$ and an octagon $(c, f)$. The diameter of the thin pins is $0.1 \mathrm{~mm}$, the length of chamfers between the two pins used in the square and the equilateral triangle cases is $0.6 \mathrm{~mm}$, and the distance from each apex of the polygonal interfaces to the geometric centre is $r_{p}=20 \mathrm{~mm}$.

pins for the equilateral triangle and the square interfaces are small (i.e. $600 \mu \mathrm{m}$ ) in order to reduce the influence of the pins on the flow field after the passage of the incident cylindrical shock wave. In two-dimensional cylindrical coordinates $(r, \theta)$, the geometric equation for a polygon can be written as

$$
r=\frac{r_{p} \cos (\pi / N)}{\cos (n \pi / N-\theta)}, \quad \frac{2(n-1) \pi}{N} \leqslant \theta \leqslant \frac{2 n \pi}{N}, \quad n=1,2, \ldots, N,
$$

where $r_{p}$ denotes the radius of the circumcircle for the polygons and $N$ stands for the number of sides of the polygonal interfaces (here $N=3,4$ and 8 , respectively). In order to ensure the same incident cylindrical shock Mach number when the shockinterface interaction happens, the value of $r_{p}$ is maintained at $20 \mathrm{~mm}$ in the three cases. Then the radius of the mean interface location $r_{i 0}$, the amplitude of the initial interface, $a_{i 0}$, and the representative wavelength of the perturbation, $\lambda_{i 0}$, can be expressed as

$$
r_{i 0}=\frac{r_{p}}{2}\left(1+\cos \frac{\pi}{N}\right), \quad a_{i 0}=\frac{r_{p}}{2}\left(1-\cos \frac{\pi}{N}\right), \quad \lambda_{i 0}=\frac{\pi r_{p}}{N}\left(1+\cos \frac{\pi}{N}\right) .
$$

As a result, the ratio between the initial amplitude and the wavelength, $a_{i 0} / \lambda_{i 0}$, can be given as

$$
\frac{a_{i 0}}{\lambda_{i 0}}=\frac{N}{2 \pi} \tan ^{2} \frac{\pi}{2 N} .
$$

For easy comparison, the values of the quantities for these initial polygonal interfaces are listed in table 1 . As the number of sides of the polygonal interfaces $(N)$ 


$\begin{array}{lccc} & \text { Equilateral triangle } & \text { Square } & \text { Octagon } \\ N & 3 & 4 & 8 \\ r_{p}(\mathrm{~mm}) & 20 & 20 & 20 \\ r_{i 0}(\mathrm{~mm}) & 15 & 17.07 & 19.24 \\ a_{i 0}(\mathrm{~mm}) & 5 & 2.93 & 0.76 \\ \lambda_{i 0}(\mathrm{~mm}) & 31.40 & 26.82 & 15.11 \\ a_{i 0} / \lambda_{i 0} & 0.16 & 0.11 & 0.05\end{array}$

TABLE 1. The values of various quantities for the three initial polygonal interfaces. $N$, the number of sides of the polygonal interfaces; $r_{p}$, the radius of the circumcircle for the polygons; $r_{i 0}$, the radius of the mean interface location; $a_{i 0}$, the amplitude of the initial interface; $\lambda_{i 0}$, the representative wavelength of the perturbation in the initial interface.

increases by keeping the radius of the circumcircle for the polygons $\left(r_{p}\right)$ constant, the radius of the mean interface locations $\left(r_{i 0}\right)$ increases, both the amplitude of the initial interfaces $\left(a_{i 0}\right)$ and the representative wavelength of the perturbation $\left(\lambda_{i 0}\right)$ decrease, and their ratio also decreases gradually.

Similar to our previous work (Wang et al. 2013), two parallel Plexiglas disks are used to maintain the shapes of the polygonal interfaces in the test section. For a certain interface shape, small holes and channels are precisely manufactured on opposite surfaces of two disks by an engraving machine in order to ensure that the geometric centre of the initial interface formed can be located at the focal point (i.e. the axis of the test section). To form an initial interface, thin pins are threaded through the holes with their ends stuck in the channels. The straight pins are perpendicular to the surfaces of the parallel Plexiglas disks, which are fixed in the test section. Prior to each experimental run, the soap film is formed by blowing a soap bubble full of the test gas (i.e. $\mathrm{SF}_{6}$ in this work) through a hole in the test section into the interface region, using an injection tube. When the soap film connects all pins, blowing of the test gas is stopped and the gas is gently pumped out to decrease the pressure inside the soap bubble. Once the soap films connecting thin pins are flat, they will be perpendicular to the surfaces of the Plexiglas disks due to the surface tension. Finally, the injection tube is taken off and the hole is sealed. In this way, the gas interface has an almost ideal two-dimensional shape with a polygonal cross-section. The effects of the thin pins and fine chamfers on the shock propagation and interface deformation have been evaluated numerically and experimentally in planar shock-polygonal interface interactions (Wang et al. 2013; Zhai et al. 2014; Luo et al. 2015), which predict a good performance of the soap film method in the interface formation and the successive RM instability study.

\subsection{Flow diagnostics}

In order to validate the feasibility and reliability of the shock tube facility for generating cylindrical shock waves, a high-speed schlieren system is used to capture the shock propagation in the test section, as shown in figure $3(a)$. The schlieren system is made up of a blade, a slit, two convex lenses, a beam splitting mirror, a schlieren concave mirror and two plane mirrors. One plane mirror is placed at the bottom of the test section and the other one is used to adjust the optical path for convenient arrangement of the instruments. Images of the flow field illuminated by a DC regulated light source (DCR III, SCHOTT North America, Inc., maximum 
(a)

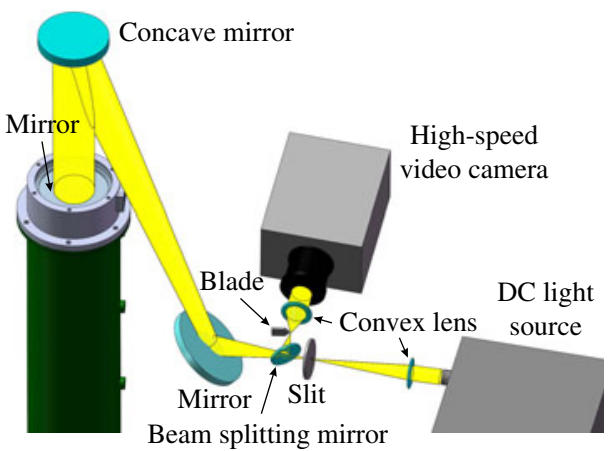

(b)

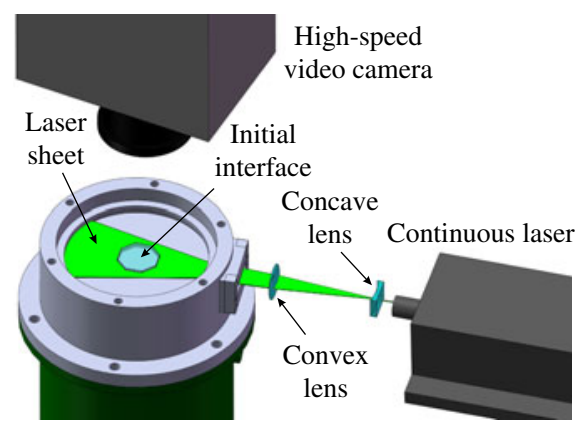

FIgURE 3. (Colour online) (a) Schematic of the high-speed schlieren system to capture the propagation of cylindrical shock waves. (b) Schematic of the laser sheet imaging system to observe the movement of the polygonal interface.

power output $200 \mathrm{~W}$ ) are captured by a high-speed video camera (FASTCAM SA5, Photron Limited, with maximum frame rate 1 million f.p.s. and maximum spatial resolution of the detector $1024 \times 1024$ pixel $^{2}$ ). The camera is connected to a computer by an ethernet interface cable and the temporal and spatial resolutions of the camera can be adjusted prior to each run. The timing and the triggering of the system are mainly controlled by a four-channel delay generator (DG645, Stanford Research Systems) combined with some auxiliary equipment.

When the initial interface is formed in the test section, the pollution of the disks by of soap liquid and the arrangement of the thin pins would contaminate the schlieren images of the evolving interface, which can be seen in studies of planar shock-polygonal interface interactions (Wang et al. 2013; Zhai et al. 2014; Luo et al. 2015). Moreover, the schlieren system often obtains an integrated view of the flow field along the light path. In this work, the observation region is small so that it is difficult to clearly identify the shock system and the boundaries of the evolving interface using high-speed schlieren photography. In order to overcome the shortcomings, planar Mie scattering photography ( $\mathrm{Si}$ et al. 2014a,b), which gives a cross-sectional view of the flow field, is implemented. A continuous high-power laser (SDL-532-15000T, Shanghai Dream Lasers technology Co. Ltd., with output $15 \mathrm{~W}$ and light wavelength $532 \mathrm{~nm}$ ) is used as the light source. An optical system consisting of a cylindrical concave lens and a convex lens is used to form a laser sheet for illuminating the flow field through transparent optical glass mounted on the sidewall of the test section, as shown in figure $3(b)$. Soap droplets from the breakup of the soap film after the incident shock passage are employed as the tracing particles (Cohen 1992; Hosseini \& Takayama 2005; Ranjan et al. 2005). The average size of the soap droplets is estimated to be about $1-10 \mu \mathrm{m}$. The laser sheet with a thickness of smaller than $1 \mathrm{~mm}$ is located through the symmetry plane of the initial interface in the middle of the test section and the high-speed video camera is placed above the test section to capture the complete evolution process of the interface.

\section{Features of cylindrical shock waves}

First of all, the generation of cylindrical converging shock waves is validated in the vertical annular diaphragmless shock tube. In an experimental run, the driver pressure and the auxiliary pressure are initially fixed to be $2.028 \times 10^{5} \mathrm{~Pa}$ and $2.5 \times 10^{5} \mathrm{~Pa}$, respectively. When the shock tube is in operation, the pressure histories in the annular 

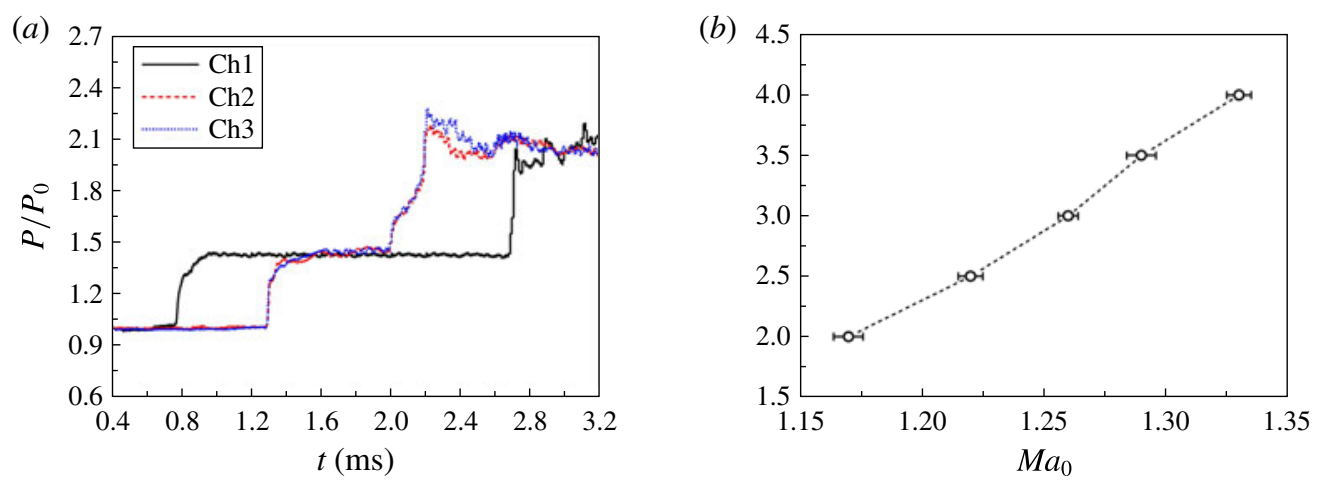

FIgure 4. (Colour online) (a) Pressure histories of transducers Ch1-Ch3 mounted on the sidewall of the driven section for $M a_{0}=1.17$. (b) Experimental measurements showing the pressure ratio between the driver and the driven sections versus the value of $M a_{0}$.

driven section are monitored by three piezoelectric pressure transducers (i.e. Ch1-Ch3 in figure 1). The position of transducer $\mathrm{Ch} 1$ is $200 \mathrm{~mm}$ lower than that of transducer $\mathrm{Ch} 2$, and transducers $\mathrm{Ch} 2$ and $\mathrm{Ch} 3$ are located at the same height, $120 \mathrm{~mm}$ from the top wall of the test section. Figure 4(a) plots the variation with time of the pressures, which are normalized by the atmospheric pressure $P_{0}=1.017 \times 10^{5} \mathrm{~Pa}$ at room temperature. The results indicate that a sudden pressure jump happens when the incident shock arrives at the transducers and the post-shock pressure keeps nearly constant at each transducer. When the shock reflects back and passes across the transducer once again, another jump arises. The good coincidence between $\mathrm{Ch} 2$ and Ch3 signals verifies the uniformly annular shock waves, as the shock wave passes over transducers $\mathrm{Ch} 2$ and $\mathrm{Ch} 3$ simultaneously. The time interval of the incident shock arrival at transducers $\mathrm{Ch} 1$ and $\mathrm{Ch} 2$ is extracted to be $518.6 \mu \mathrm{s}$. Therefore, the velocity of the incident annular shock wave in the driven section can be calculated to be $385.7 \mathrm{~m} \mathrm{~s}^{-1}$, corresponding to a Mach number of $M a_{0}=1.17$. It should be mentioned that different values of the shock Mach number can be obtained by changing the driver pressure. Figure $4(b)$ presents the pressure ratio, $P / P_{0}$, between the driver and driven sections versus the shock Mach number, $M a_{0}$, in experiments. The value of $M a_{0}$ increases monotonically as the driver pressure increases. For a fixed driver pressure, several runs of the experiments are conducted and nearly the same shock Mach number can be achieved, which verifies the high reproducibility of the vertical shock tube facility.

In order to track the shock front in experiments, the frame rate of the camera is adjusted up to 105000 f.p.s., corresponding to a time interval between two consecutive frames of about $9.52 \mu \mathrm{s}$, and the shutter of the camera is set at $1 \mu \mathrm{s}$. Figure 5 shows schlieren images of the cylindrical shock waves propagating in the converging process for $M a_{0}=1.17$. In this experiment, the spatial resolution of the camera is about $0.39 \mathrm{~mm} \mathrm{pixel}^{-1}$ (i.e. the image height is 192 pixels, corresponding to a real value of $74.9 \mathrm{~mm}$ ). Therefore, the motion blur can be maintained within 2 pixels (i.e. $0.78 \mathrm{~mm}$ ) for a shock wave with velocity of less than $780 \mathrm{~m} \mathrm{~s}^{-1}$. In the observation region, the cylindrical shock wave has already steepened with propagation. The shock front matches well with the circular arc whose centre is located at the axis of the test section, which indicates that the cylindrical shock waves are uniformly formed in perfectly two-dimensional circular shapes. 

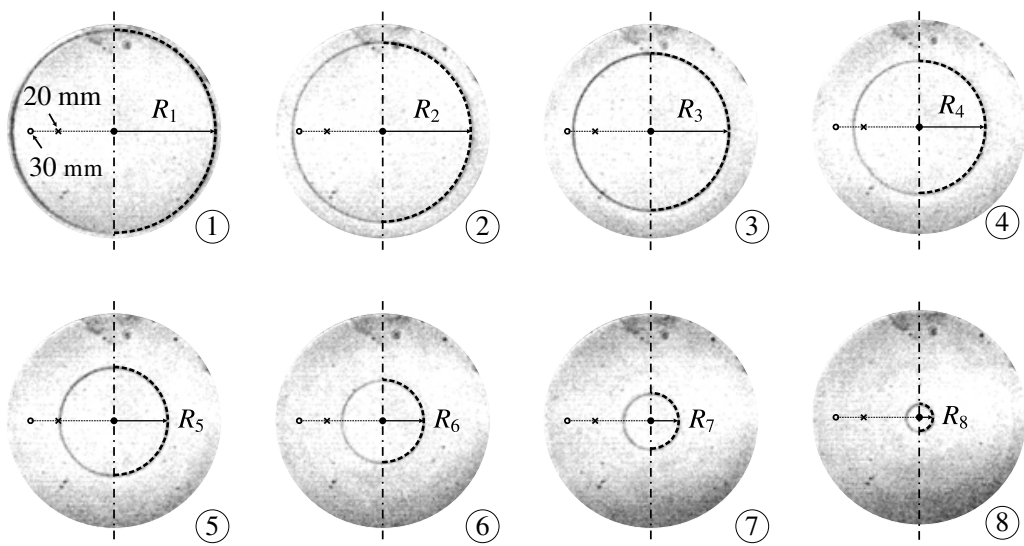

FIGURE 5. Sequence of schlieren images showing the propagation of cylindrical shock waves in the converging process for $M a_{0}=1.17$. The dashed line on the right half of each image represents a circular arc whose centre is located at the focal point. $R_{i}(i=1-8)$ stands for the radius of the cylindrical shock front. The frame rate of the high-speed video camera is 105000 f.p.s. corresponding to a time interval between two consecutive frames of about $9.52 \mu \mathrm{s}$. The number in the lower right corner of each image represents the time sequence. The locations of 20 and $30 \mathrm{~mm}$ from the focal point are also marked.
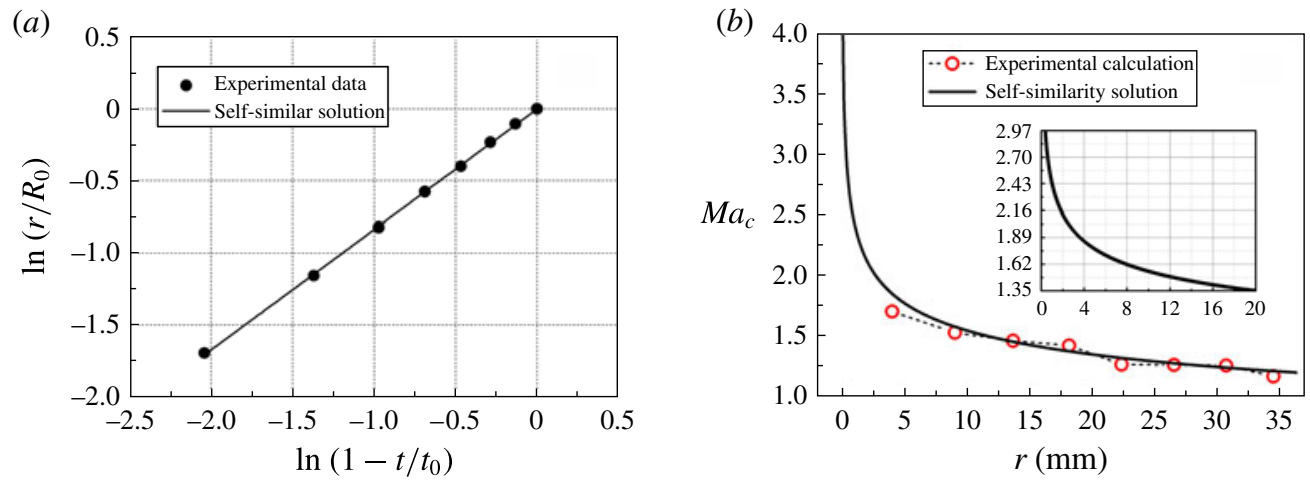

FIGURE 6. (Colour online) (a) Logarithmic plot of the shock position as a function of time with a fitted line of the self-similarity solution for $M a_{0}=1.17$. (b) Experimental measurements and self-similarity fitted line showing the variation of shock Mach numbers as a function of the position. The inset shows a magnification of the fitted line within $r=20 \mathrm{~mm}$.

The radii of the cylindrical shock fronts, $R_{i}(i=1-8)$, at different times can be measured from figure 5 and the variation of shock positions versus time can be obtained, as plotted in figure $6(a)$ in logarithmic scales. Guderley (1942) first analytically studied the propagation of converging shock waves and presented a self-similarity solution regarding the shock radius $r$ as a function of time $t$ in the form

$$
\frac{r}{R_{0}}=\left(1-\frac{t}{t_{0}}\right)^{\alpha},
$$



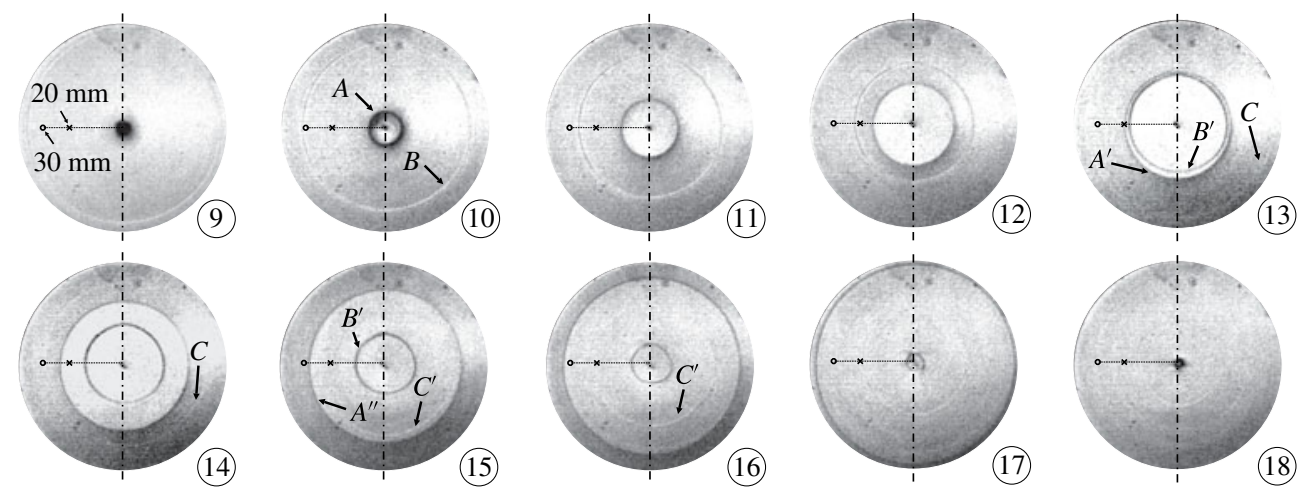

FIGURE 7. Sequence of schlieren images showing the propagation of shock waves in observation region for $M a_{0}=1.17$, which is a continuation of figure 5. Again, the frame rate of the high-speed video camera is 105000 f.p.s. corresponding to a time interval between two consecutive frames of about $9.52 \mu \mathrm{s}$. The number in the lower right corner of each image represents the sequence of the images. The locations of 20 and $30 \mathrm{~mm}$ away from the focal point are marked.

where $R_{0}$ and $t_{0}$ represent the initial radius of the converging shock location at $t=0$ and the time interval as the shock arrives at the focal point from its initial location, respectively. It was found that the similarity constant $\alpha$ was about 0.835 for a strong cylindrical converging shock wave in air (with adiabatic exponent $\gamma=1.4$ ). Later, several experiments (Matsuo \& Nakamura 1980, 1981; Takayama et al. 1987; Baronets 1994; Hosseini \& Takayama 2010; Kjellander et al. 2011) were performed to determine the value of $\alpha$ with different incident Mach numbers and various adiabatic exponents of gases. The results indicated that the value of $\alpha$ was heavily dependent on the adiabatic exponent and could remain stable in a wide range of incident shock Mach numbers. In this experiment, $R_{0}=36.28 \mathrm{~mm}$ is taken as the radius $R_{1}$ of the outmost shock front and $t_{0}=76.63 \mu \mathrm{s}$ is calculated from the experimental data in figure $6(a)$ using the fitting of least squares. A fitting similarity constant $\alpha=0.836 \pm 0.005$ is obtained. The good agreement of the experimental observation with previous experimental and analytical results verifies the reliability of the shock tube facility.

The shock Mach numbers of the cylindrical shock, $M a_{c}$, changing with the position, $r$, in the converging process can be obtained by calculating the velocity of the cylindrical shock fronts, i.e.

$$
M a_{c}(r)=\frac{1}{a_{0}} \frac{\mathrm{d} r}{\mathrm{~d} t}=\frac{1}{a_{0}} \frac{\alpha R_{0}}{t_{0}}\left(\frac{r}{R_{0}}\right)^{(\alpha-1) / \alpha},
$$

where $a_{0}=329 \mathrm{~m} \mathrm{~s}^{-1}$ is the local sound speed. Figure $6(b)$ plots the variations with radius of the cylindrical shock Mach numbers predicted by (3.2) as well as the experimental evaluation, where a good agreement between them is achieved. From (3.2), the Mach number of the incident cylindrical shock propagating in the test section can be easily obtained (e.g. the shock Mach number at $r=20 \mathrm{~mm}$ is about 1.35).

In this vertical shock tube, the curved wall in the corner connecting the driven section and the test section usually induces disturbances in the flow after the arrival 


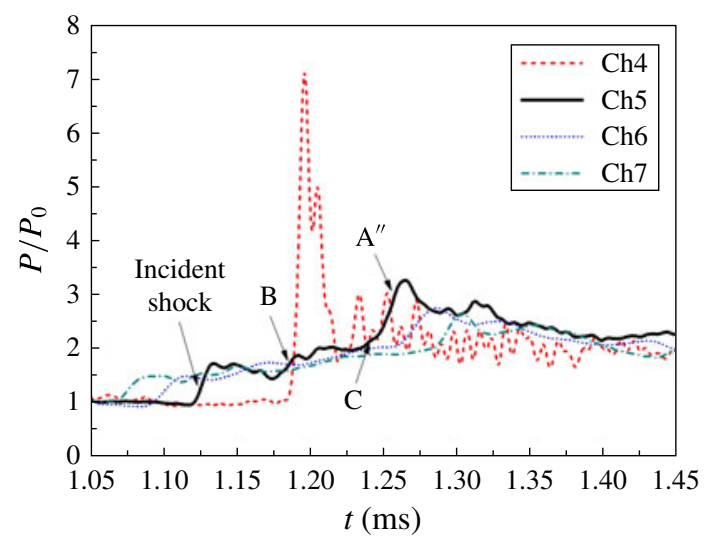

FIGURE 8. (Colour online) Experimental pressure histories at transducers Ch4-Ch7 mounted on the top wall of the test section. The arrival of different shock waves at transducer Ch5 (30 mm from the focal point, as indicated in figure 7) are highlighted.

of the incident shock wave. Figure 7 shows a continuation of the sequence of schlieren images presented in figure 5. In order to match the information on time, pressure and shape for the shock propagation, four piezoelectric pressure transducers (i.e. Ch4-Ch7 in figure 1), mounted on the top wall of the test section, are used to obtain the pressure variation with time. The Ch4 transducer is located at the centre of convergence (i.e. the axis of the test section) and the $\mathrm{Ch} 5-\mathrm{Ch} 7$ transducers are located away from the focal point at radii of $30 \mathrm{~mm}, 40 \mathrm{~mm}$ and $50 \mathrm{~mm}$, respectively. As shown in figure 8, the incident shock passes across each transducer in quick succession, resulting in a pressure jump at each transducer position. The post-shock pressure increases as the incident shock propagates towards the focal point. When the incident shock arrives at the Ch4 transducer located at the focal point, the post-shock pressure reaches a maximum value. When the reflected shock comes back, another pressure jump arises and the post-shock pressure decreases gradually, with some fluctuations at each position of the transducers. In particular, the signal at the Ch5 transducer (30 mm away from the focal point) is highlighted on the figure. At the beginning, the incident cylindrical shock wave with a circular shape converges to the focal point. When the reflected shock (shock-A) arises, the first disturbed shock wave (shock-B) appears. Shock-B is weak so that the pressure jump is relatively low when shock-B passes across the Ch5 transducer. Then shock-A interacts with shock-B, with one exploding (shock- $\mathrm{A}^{\prime}$ ) and the other one imploding (shock- $\mathrm{B}^{\prime}$ ). Meanwhile, another weak shock wave (shock-C) moves to the observation region and quickly collides with shock- $\mathrm{A}^{\prime}$, resulting in two new shock waves (shock- $\mathrm{A}^{\prime \prime}$ and shock-C'). Only several microseconds later, shock- $\mathrm{A}^{\prime \prime}$ arrives at the Ch5 transducer and a sharp pressure jump arises. As time passes, shock- $\mathrm{B}^{\prime}$ moves to the centre of convergence and some more disturbances appear in the flow. It is worth mentioning that most disturbances are weak and thus have little influence on the strength and morphology of primary cylindrical shock waves, i.e. shock-A series.

\section{Evolution of the polygonal interface}

Three sets of experimental runs in the case of an air/SF 6 (fast/slow) configuration corresponding to the three polygonal interfaces have been conducted. The Atwood 

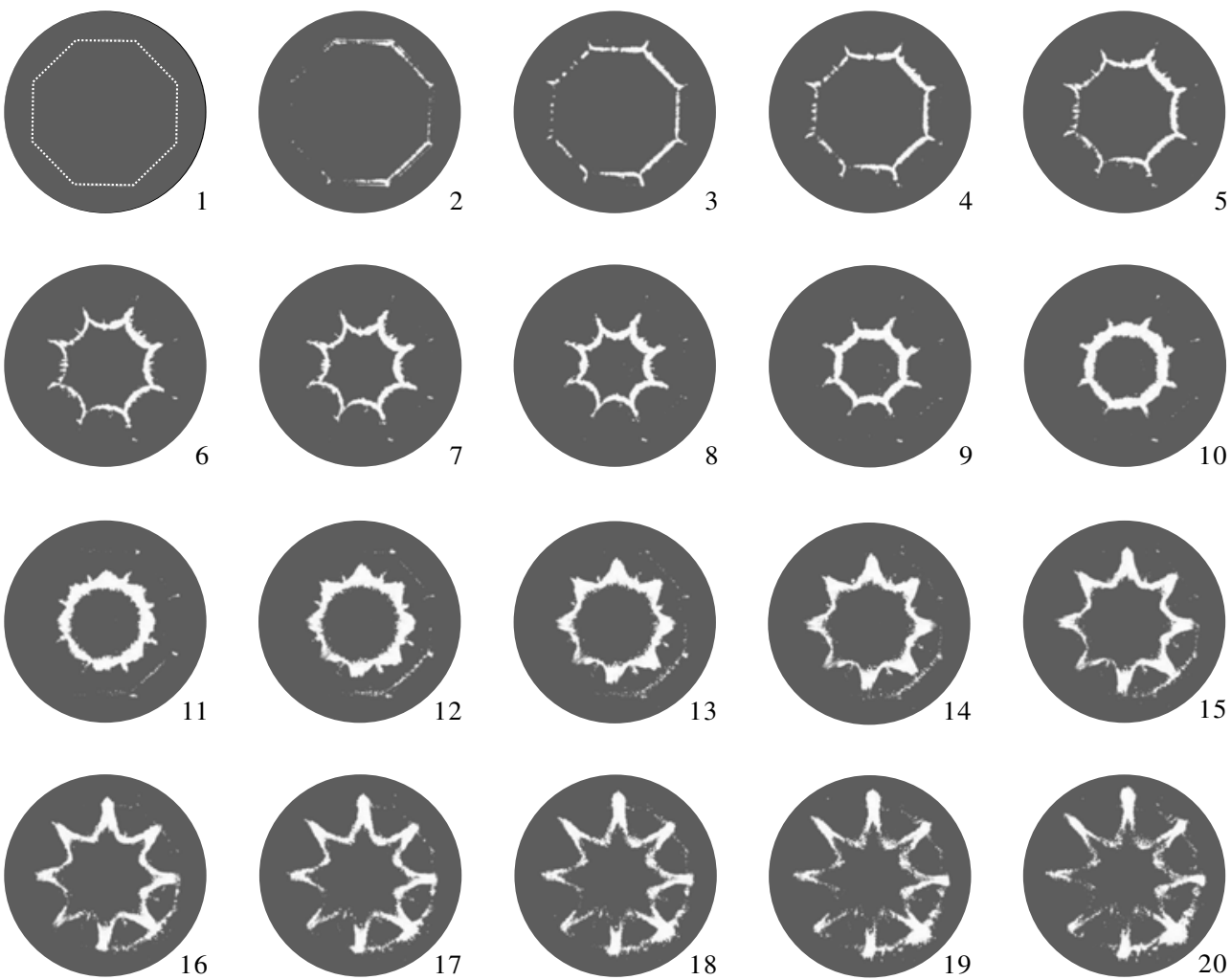

FIGURE 9. Sequence of cross-sectional views of the octagonal interface accelerated by the incident cylindrical shock and the reflected shock. The frame rate of the camera is 62500 f.p.s. corresponding to a time interval between two consecutive frames of $16 \mu \mathrm{s}$. The number in the lower right corner of each image represents the time sequence of the frames. The initial interface is highlighted in frame 1 by a dotted line.

number, $A_{t}$, is defined as

$$
A_{t}=\frac{\rho_{1}-\rho_{2}}{\rho_{1}+\rho_{2}}
$$

where $\rho_{1}$ represents the gas density inside the soap bubble and $\rho_{2}$ is the gas density outside the soap bubble. The density of pure $\mathrm{SF}_{6}$ is $\rho_{1}=5.97 \mathrm{~kg} \mathrm{~m}^{-3}$ and the density of air is $\rho_{2}=1.19 \mathrm{~kg} \mathrm{~m}^{-3}$. If the contamination by air of $\mathrm{SF}_{6}$ inside the volume and the contamination by $\mathrm{SF}_{6}$ of air outside the volume can be neglected, the Atwood number is calculated to be $A_{t}=0.699$. Representative experimental results showing the evolution of the polygonal gas interfaces during the early stage of RM instability as well as constructed schematics of the interface deformation and the shock propagation are given in figures 9-14. In experiments, the frame rate of the camera is chosen as 62500 f.p.s. corresponding to a time interval between two consecutive frames of 16 $\mu \mathrm{s}$. The spatial resolution is $320 \times 320$ pixel $^{2}$ and the shutter rate of the camera is set at $1 / 517000 \mathrm{~s}$. In this situation, the spatial resolution in each experimental run is about $0.23 \mathrm{~mm} \mathrm{pixel}{ }^{-1}$. Therefore, the motion blur in each image is limited within 1 pixel for an interface velocity of below $120 \mathrm{~m} \mathrm{~s}^{-1}$. Note that for convenience of measurements, each original square frame in experiments has been cut into a circular area with its centre coinciding with the geometric centre of the initial interface. 


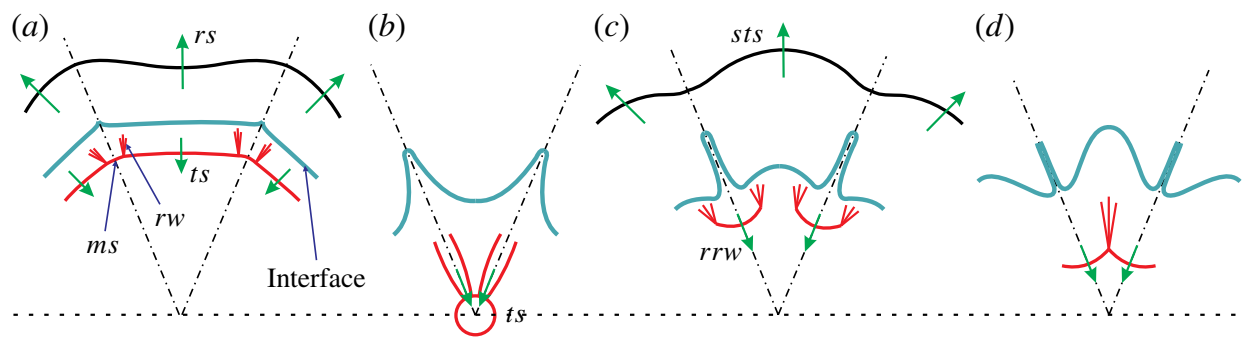

FIGURE 10. (Colour online) Schematics of the interface deformation and the shock propagation for the octagonal gas cylinder: $(a)$ the incident cylindrical shock passes across the interface; $(b)$ the transmitted shock converges to the centre; $(c)$ the reflected shock impacts on the evolving interface; $(d)$ the interface develops further. Here $t s$, transmitted shock; $r s$, reflected shock; $m s$, Mach stem; $r w$, rarefaction waves; sts, second transmitted shock; rrw, reflected rarefaction waves.
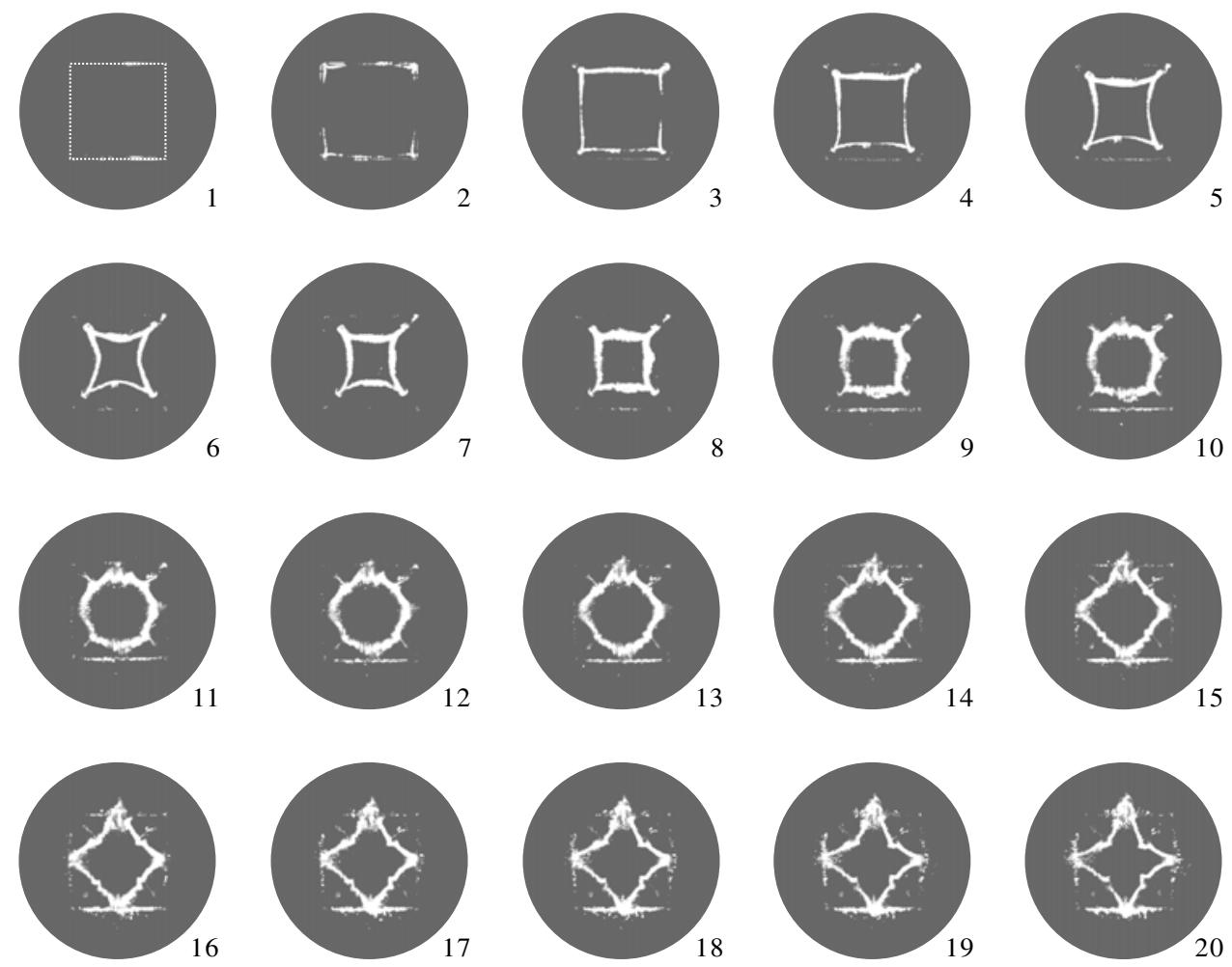

FIGURE 11. Sequence of cross-sectional views of the square interface accelerated by the incident cylindrical shock and the reflected shock. The frame rate of the camera is 62500 f.p.s. corresponding to a time interval between two consecutive frames of $16 \mu s$. The number in the lower right corner of each image represents the time sequence of the frames. The initial interface is highlighted in frame 1 by a dotted line.

Moreover, the contrast and the brightness of experimental images have been slightly adjusted for a better view of the background by maintaining the same morphologies of the distorted interfaces as those in the original dynamic images. 
(a)

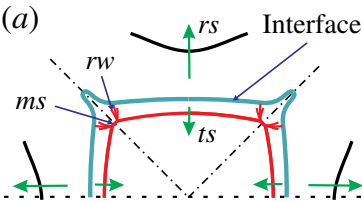

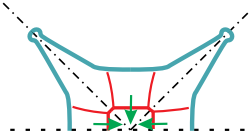

(c)

(d)

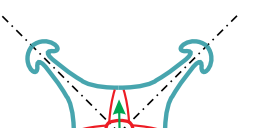

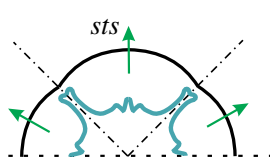

FIgURE 12. (Colour online) Schematics of the interface deformation and the shock propagation for the square gas cylinder: $(a)$ the incident cylindrical shock passes across the interface; $(b)$ the transmitted shock moves to the focal point; $(c)$ the transmitted shock is reflected; $(d)$ the reflected shock impacts the evolving interface. The symbols are the same as in figure 10.
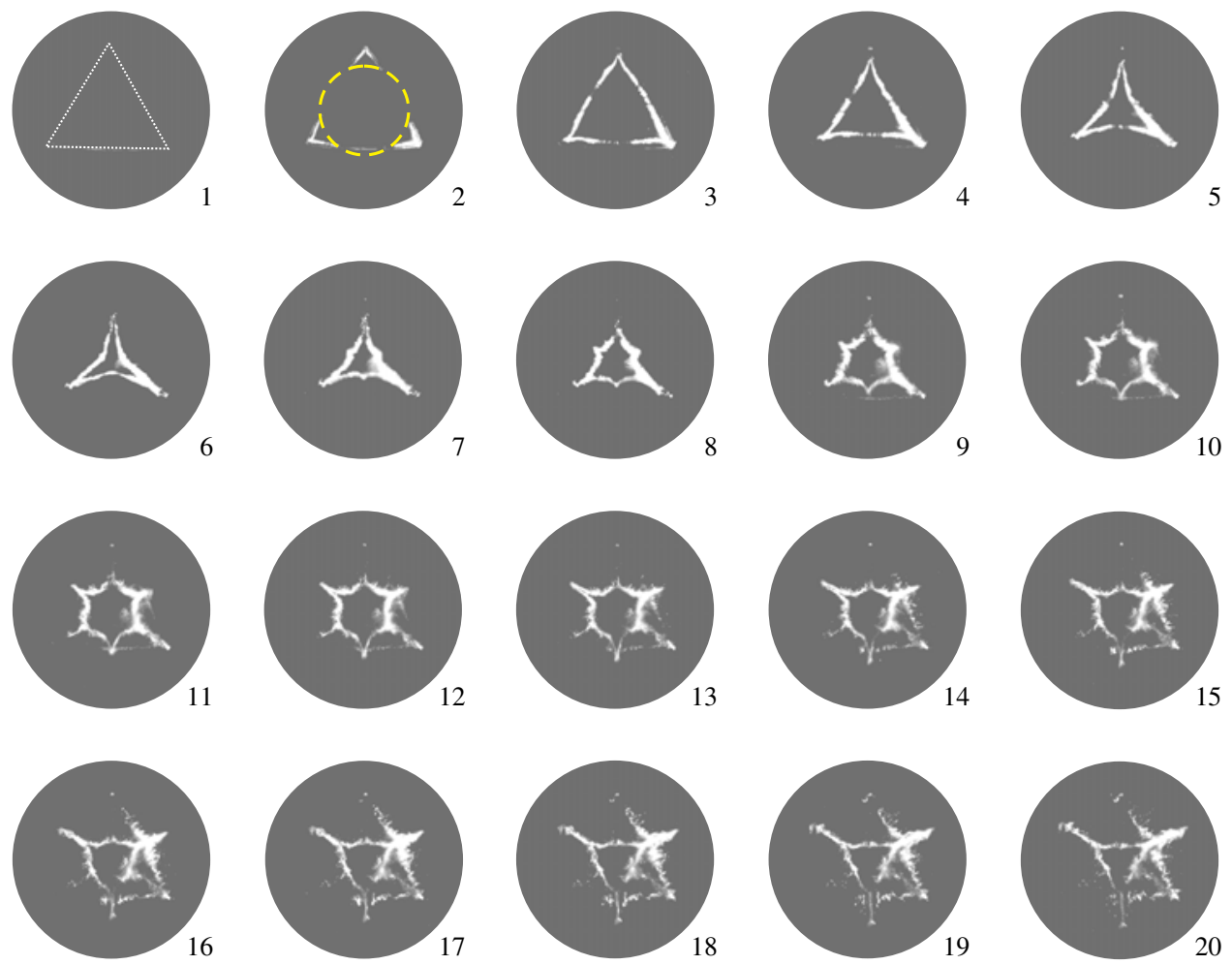

FIgURE 13. (Colour online) Sequence of cross-sectional views of the equilateral triangle interface accelerated by the incident cylindrical shock and the reflected shock. The frame rate of the camera is 62500 f.p.s. corresponding to a time interval between two consecutive frames of $16 \mu \mathrm{s}$. The number in the lower right corner of each image represents the time sequence of the frames. The initial interface is highlighted in frame 1 by a dotted line. The position of the incident cylindrical shock assuming there is no interface is sketched in frame 2 by a dashed line.

When the incident cylindrical shock initially arrives at the apexes of the polygonal interface $(r=20 \mathrm{~mm})$, the instantaneous shock Mach number obtained from equation (3.2) is about 1.35. Then the incident shock passes across the interface, inducing an irregular refraction. The type of the irregular refraction is heavily dependent on the inclined angle, defined as the angle between the boundaries of the interface and 


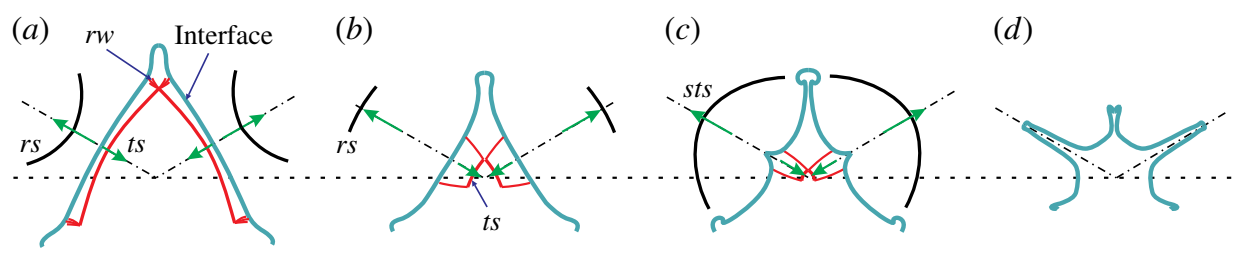

FIGURE 14. (Colour online) Schematics of the interface deformation and the shock propagation for the equilateral triangular gas cylinder: $(a)$ the incident cylindrical shock passes across the interface; $(b)$ the transmitted shock moves to the focal point; $(c)$ the reflected shock impacts the evolving interface; $(d)$ the interface further develops. The symbols are the same as in figure 10 .

the incident shock wave. For a planar shock with a fixed Mach number interacting with a polygonal interface, the inclined angle remains constant at the upstream and downstream interfaces, respectively, and thus the irregular refraction system can be identified (Zhai et al. 2014; Luo et al. 2015). However, for a cylindrical shock case, the inclined angle always changes as the incident shock moves along the sides of the interface, and the irregular refraction system will change as well. For example, as the cylindrical shock passes across the interface from the apex to the side centre of the interface, the inclined angle changes from $22.5^{\circ}$ to $0^{\circ}$ in the octagon case, from $45^{\circ}$ to $0^{\circ}$ in the square case, and from $60^{\circ}$ to $0^{\circ}$ in the equilateral triangle case. Furthermore, due to the geometric convergence, the strength of the cylindrical shock at the external interface will increase as the shock moves from the apex to the side centre of the interface. As a result, the phenomena reported here will be distinct from those in planar shock cases.

Figure 9 presents a sequence of a cross-sectional view of the octagonal gas cylinder accelerated by the incident cylindrical shock and the reflected shock. The shape of the initial interface is presented in frame 1, which has been strengthened by dashed lines because the light scattering of the thin soap film initially separating $\mathrm{SF}_{6}$ from atmospheric air is very weak. When the incident converging shock impacts on the interface, the soap film is atomized and the Mie scattering light of the soap liquid droplets can be captured by the camera. As the propagation of shock waves is invisible in these images, a schematic of the interface deformation and the shock propagation was constructed based on the experimental results and shock dynamics theory, as shown in figure 10.

At the beginning, the incident cylindrical shock impacts on the octagonal interface, resulting in a 'slow' transmitted shock $(t s)$ and a 'fast' reflected shock $(r s)$ because of the larger acoustic impedance of $\mathrm{SF}_{6}$ inside the volume (figure 10a). Shock $r s$ propagates outwards with a wavy configuration, in which each crest (trough) comes from the interaction of the cylindrical shock with each apex (side centre) of the interface. The inward-moving $t s$ has an approximately octagonal shape, where rarefaction waves $(r w)$ and a Mach stem $(m s)$ connected by a pair of triple points in the vicinity of each apex of the interface will appear due to the small inclined angles in the shock-interface interaction.

Meanwhile, the eight apexes of the octagonal interface turn into spikes from $\mathrm{SF}_{6}$ to air, and the eight sides of the octagon interface become curved and develop into bubbles from air to $\mathrm{SF}_{6}$, as shown in figure 9 (frames 2-8). In this period, $r w$ propagates behind $t s$ and they merge gradually (figure 10b). Because each pair of triple points originates from one apex and will converge to the focal point, the 
trajectory of each triple point at the $t s$ front will be a curved line, which is different from the planar case (the triple point travels along almost a straight line as indicated in Luo et al. 2015). As time proceeds, the focusing of $t s$ occurs within the volume, inducing high pressure and density in the focal point. Shock $t s$ reflects from the focal point, interacts with the other waves and finally impacts on the evolving interface. This secondary interaction happens at about $120 \mu \mathrm{s}$ after the incident shock reaches the octagonal interface (frame 9), resulting in a secondary transmitted shock (sts) and reflected rarefaction waves ( $r r w$ ) (figure 10c). In this process, a reversal of the evolving interface can be formed. The bubbles from air to $\mathrm{SF}_{6}$ begin moving in an opposite direction and develop into new spikes from $\mathrm{SF}_{6}$ to air; the spikes suddenly stop moving, and subsequently, they are swallowed up by the bulk interface, forming new bubbles from air to $\mathrm{SF}_{6}$ (frames 10-14). Both the spikes and the bubbles of the evolving interface move outwards. Because the evolving interface and the shock waves within the volume are irregular, the complex interaction and merging of rrw will take place (figure $10 d$ ).

It should be noted that a secondary, inward-facing shock forms at the tail of the rrw (Lombardini et al. 2014a). This only appears in spherical and cylindrical flows, and does not arise in planar geometries such as flows in a constant-cross-section shock tube with an endwall. Unfortunately, this secondary shock cannot be observed by the planar Mie scattering photography used. However, because it is expected that this secondary shock will cause progressively weaker reshocks on the interface, the evolution of the interface will exhibit some extraordinary features which could be captured by the laser sheet photography. Later on, the bubbles start moving inwards (frames 16-20) and the whole interface continues growing in time. The disturbed shock waves appearing in figure 7 also interact with the evolving interface and the substantial waves in the process. They are much weaker than the incident shock (A series in figure 7) at the same position, as indicated in figure 8, so that their effect on the evolution of the interface would be insignificant in comparison with the primary shock waves.

For the square case, the experimental results and corresponding constructed wave pattern and interface structures are presented in figures 11 and 12, respectively. An irregular refraction can be found after the passage of the incident cylindrical shock. Shock $r s$ has a wavy shape with a very large amplitude because the formation of the wave crests is much earlier than that of the troughs. As $r s$ moves outwards, its crests expand and it becomes very weak, while the troughs remain significant (figure 12a). The structure of $t s$ is similar to that in the octagonal case, but the triple points follow different curves because the inclined angles and the shape of $t s$ are different from those in the octagonal case. Accordingly, the four apexes of the interface become spikes from $\mathrm{SF}_{6}$ to air, as shown in figure 11 (frames 2-3). Shock ts propagates towards the focal point and reflects back, resulting in the merging and the interaction of the following $r w$ within the volume (figure $12 b, c$ ). During this period, the sides of the interface develop into bubbles moving from air to $\mathrm{SF}_{6}$ and small mushroom structures arise in the spikes (frames 4-7). After the reflected shock passes across the evolving interface, complex wave patterns are formed, and a reversal of the evolving interface occurs (figure $12 d$ ). New spikes from $\mathrm{SF}_{6}$ to air form at the side centre (frames 8-10), and the original angular interfaces disappear gradually while new bubbles from air to $\mathrm{SF}_{6}$ develop (frames 11-15). As time proceeds, the amplitudes of the disturbances on the spikes and the bubbles increase. The spikes develop outwards while the bubbles move inwards (frames 16-20). In the process, the reflection and transmission of different shock waves continuously promote the interface development. 
In the equilateral triangular case, the distance from each apex to the geometric centre (i.e. $r=20 \mathrm{~mm}$ ) is twice as long as that from each side centre to the geometric centre (i.e. $r=10 \mathrm{~mm}$ ). Figure 13 presents the deformation of the equilateral triangular interface with a time interval between two consecutive frames of $16 \mu$ s. Figure 14 gives the corresponding constructed schematic of the interface deformation and the shock propagation. As the shocked interface is bright in comparison with the dark initial interface, the incident cylindrical shock can be recognized in frame 2 of figure 13 and a schematic of its position without the interface is sketched by a yellow dashed line. In this case, the inclined angle is relatively large at the beginning, and thus $r s$ would be divided into three parts once it is formed (figure 14a). The three branches of $r s$ move with its shape curving from inwards to outwards, and $t s$ converges gradually to the focal point (figure $14 b$ ). Shock $t s$ has nearly the same structure as before, but the length of $m s$ will be very small. In this period the interface is compressed to a very small region (frames 3-6). As the reflected shock interacts with the evolving interface, disturbances with sharp vertexes emerge on the boundaries of the bubbles because of the formation of sts and rrw (figure 14c). Then the volume of the evolving interface becomes enlarged (frames 7-11). The original angular interfaces stop moving inwards and new spikes grow (figure 14d). The cross-sectional shape of the evolving interface changes from a hexagon to an inverted triangle (frames 12-15). Later on, the mass of $\mathrm{SF}_{6}$ transfers from the main body to three spikes, resulting in promotion of the reversal of the evolving interface (frames 16-20).

\section{Vorticity generation and circulation deposition}

When a shock wave passes across an initially perturbed interface, vorticity will be generated in the flow field, governed by the vorticity transport equation:

$$
\frac{\mathrm{D} \boldsymbol{\omega}}{\mathrm{D} t}=\frac{\nabla \rho \times \nabla p}{\rho^{2}}+(\boldsymbol{\omega} \cdot \nabla) \boldsymbol{u}-\boldsymbol{\omega}(\boldsymbol{\nabla} \cdot \boldsymbol{u})+v \nabla^{2} \boldsymbol{\omega},
$$

where $\boldsymbol{\omega}, \boldsymbol{u}, v, \nabla \rho$ and $\nabla p$ denote the vorticity vector, the velocity vector, the viscosity coefficient, and the density and pressure gradients, respectively. The baroclinic term, $(\nabla \rho \times \nabla p) / \rho^{2}$, represents the vorticity generation due to the misalignment between the density and pressure gradients, which is considered as the most significant factor influencing the RM instability at weak shock strengths (Brouillette 2002; Ranjan et al. 2011). The vortex stretching term, $(\boldsymbol{\omega} \cdot \boldsymbol{\nabla}) \boldsymbol{u}$, occurs when the velocity fields are inhomogeneous, especially for a three-dimensionally developing gas interface. The vortex dilatation term, $\boldsymbol{\omega}(\boldsymbol{\nabla} \cdot \boldsymbol{u})$, is mainly caused by the gas compressibility due to high shock strengths. The viscous term, $v \nabla^{2} \omega$, stands for the vorticity generation due to the viscous effects in the flow.

In this work, the viscous diffusion can be neglected because of the low viscosity of the gases and very short duration of the experiments. The shock strengths are relatively weak (e.g. the incident cylindrical shock Mach number is 1.35 when the shock arrives at the interface) so that the vortex dilatation is relatively small. The three-dimensional behaviours of the evolving interface are also insignificant in the three cases considered here, especially in the early stage. Therefore, the deformation of the different polygonal gas cylinders induced by cylindrical shock waves is mainly ascribed to the baroclinic vorticity. Figure 15 illustrates representative schematics of the baroclinic vorticity generation for the three $\mathrm{SF}_{6}$ interfaces (octagon, square and equilateral triangle). After the passage of the incident cylindrical shock, vorticity is 
(a)

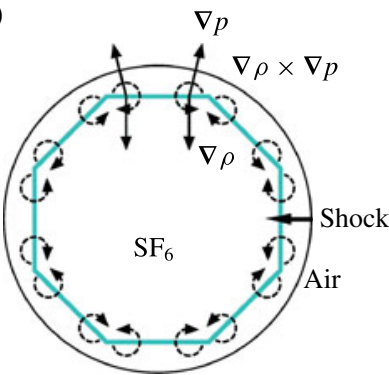

(d)

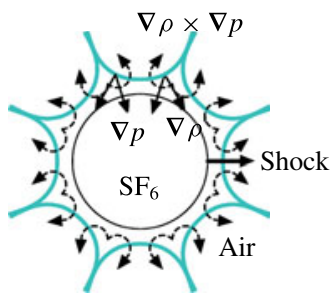

(b)

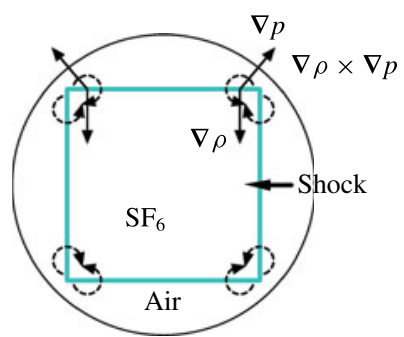

(e)

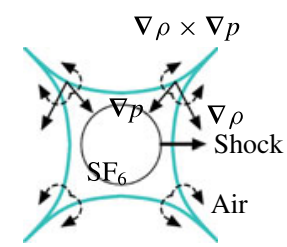

(c)

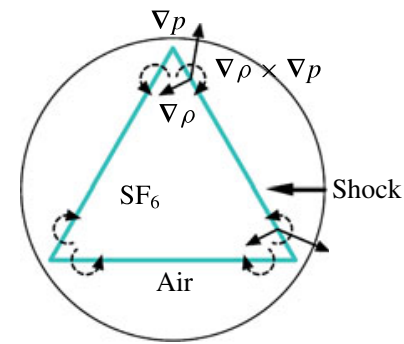

$(f)$

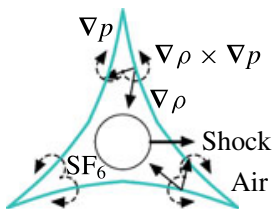

FIgURE 15. (Colour online) Representative schematics of the polygonal gas cylinder with cross-sections of $(a, d)$ an octagon, $(b, e)$ a square and $(c, f)$ an equilateral triangle, interacting with the incident cylindrical shock $(a-c)$ and the reflected shock $(d-f)$, showing the baroclinic vorticity generation. In each image the interface boundaries are simplified to be curved lines and the reflected shock is shown as a circular ring. The pressure gradients due to the effects of the converging shock geometry in the flow are not presented.

generated and mainly distributed initially on the interface surface where the density gradient exists. As time proceeds, the vorticity distribution changes and the gas interface develops. When the reflected shock arrives, additional vorticity will be deposited on the evolving interface, resulting in further interface deformation.

In a study of shock-accelerated density-stratified interfaces, Samtaney \& Zabusky (1994) provided a scaling law analytically for the baroclinic vorticity generation in RM environments based on the shock polar analysis. The normalized circulation, $\Gamma$, deposited per unit length of the planar fast-slow gas interface can be expressed to the lowest order as

$$
\Gamma=\frac{2 \gamma^{1 / 2}}{\gamma+1}\left(1-\eta^{-1 / 2}\right)\left(1+M a^{-1}+2 M a^{-2}\right)(M a-1) \sin (\phi),
$$

where $M a$ is the incident shock Mach number, $\eta$ is the density ratio of the gases on each side of the interface, $\gamma$ is the ratio of specific heat for gases and $\phi$ is the inclined angle between the shock and the interface. The normalized circulation can be converted to physical units by multiplying by the ratio of the sound speed to $\gamma^{1 / 2}$ (Samtaney \& Zabusky 1994). In this work, we assume that in an extremely small region the cylindrical shock could be considered as a planar one. Therefore, the expression for normalized circulation, $\Gamma$, initially deposited per unit length along the fast-slow polygonal interface in the case of a cylindrical converging shock wave, can be given as

$$
\Gamma=\frac{2 \gamma^{1 / 2}}{\gamma+1}\left(1-\eta^{-1 / 2}\right)\left(1+M a_{c}^{-1}+2 M a_{c}^{-2}\right)\left(M a_{c}-1\right) \sin (\phi),
$$




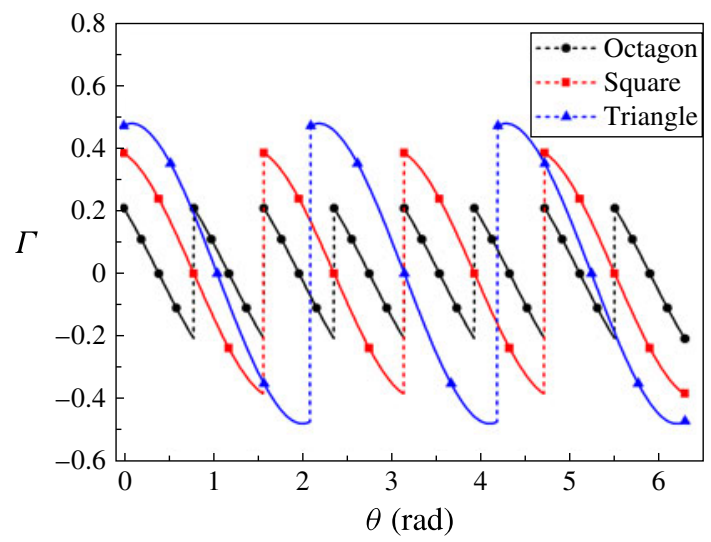

FIGURE 16. (Colour online) Normalized circulation $\Gamma$ deposited per unit length along the initial interface boundaries for cross-sections of an octagon, a square and an equilateral triangle. The circulation is normalized by a constant which has a unit of velocity, i.e. $a_{0} / \sqrt{\gamma}=278 \mathrm{~m} \mathrm{~s}^{-1}$.

$$
\begin{gathered}
M a_{c}=\frac{1}{a_{0}} \frac{\alpha R_{0}}{t_{0}}\left[\frac{r_{p}}{R_{0}} \frac{\cos (\pi / N)}{\cos (n \pi / N-\theta)}\right]^{(\alpha-1) / \alpha}, \\
\phi=\frac{(2 n-1) \pi}{N}-\theta, \quad \frac{2(n-1) \pi}{N} \leqslant \theta \leqslant \frac{2 n \pi}{N}, \quad n=1,2, \ldots, N .
\end{gathered}
$$

Here the inclined angle $\phi$ between the shock and the initial interface follows $-\pi / N \leqslant \phi \leqslant \pi / N$, and the sign of $\phi$ determines the direction of vorticity (the counter-clockwise direction is positive). When the incident cylindrical shock impacts on the polygonal gas cylinder, the pressure gradients at different points will change because the shock strength is gradually increasing as the incident cylindrical shock moves from the apex to the side centre of the polygonal gas cylinder. Figure 16 presents the values of normalized circulation, $\Gamma$, initially deposited along the gas interface for cross-sections of an octagon $(N=8)$, a square $(N=4)$ and an equilateral triangle $(N=3)$, respectively. The normalized circulations at different points in a period are obtained at different instants. In this work, the values of quantities in (5.3) are $\gamma=1.40, \eta=\rho_{1} / \rho_{2}=5.02, a_{0}=329 \mathrm{~m} \mathrm{~s}^{-1}, r_{p}=20 \mathrm{~mm}, R_{0}=36.28 \mathrm{~mm}$, $t_{0}=76.63 \mu \mathrm{s}$ and $\alpha=0.836$. As the circulation is periodically distributed along the initial interface, the total positive circulation, $\Gamma^{*}$, deposited on each interface can be expressed as

$$
\Gamma^{*}=N \int_{0}^{\pi / N} \frac{a_{0}}{\sqrt{\gamma}} \Gamma r \mathrm{~d} \theta=\frac{a_{0} N}{\sqrt{\gamma}} \int_{0}^{\pi / N} \Gamma r \mathrm{~d} \theta .
$$

As indicated in figure 16, the theoretical value of the positive normalized circulation around the apexes of the initial interface decreases from the equilateral triangular, to the square and to the octagonal interfaces. Therefore, the equilateral triangular apexes of the interface will have the highest velocity, and the octagonal one will have the lowest velocity among the three cases. It is interesting that the maximum circulation appears around the apexes for the square and the octagonal interfaces while it is away from the apexes for the equilateral triangular interface. Therefore, the trend of the velocities changing with time for the equilateral triangular interface 
will be different from the other cases at the very beginning. Quantitatively, the values of $\Gamma^{*}$ are calculated by (5.4) to be $3.73,3.25$ and $1.81 \mathrm{~m}^{2} \mathrm{~s}^{-1}$ for the equilateral triangular, the square and the octagonal interfaces, respectively. The differences of the circulation deposition for the three polygonal interfaces will result in different interface velocities and perturbation growth rates.

\section{Quantitative analysis of the interface features}

\subsection{Coordinate transformation}

To quantitatively analyse the development of the shocked polygonal interfaces, we further measure the interface structural features, including the displacements of the apex and the side centre, and the amplitudes of the perturbations in the initial interfaces. The velocities of the interfaces and the growth rates of perturbations are also calculated. For convenience, a two-dimensional transformation from cylindrical coordinates $(r, \theta)$ to Cartesian coordinates $(x, y)$ is first performed. The computational region of each experimental image is arranged to be a circular area with its centre coinciding with the geometric centre of the initial interface. Therefore, the coordinate transformation can be written as

$$
\begin{aligned}
& y=r, \quad 0 \leqslant r \leqslant r_{0}, \quad 0 \leqslant y \leqslant y_{0}, \\
& \frac{x}{x_{0}}=\frac{\theta}{2 \pi}, \quad 0 \leqslant \theta \leqslant 2 \pi, \quad 0 \leqslant x \leqslant x_{0},
\end{aligned}
$$

where $r_{0}, x_{0}$ and $y_{0}$ are the radius of the outermost boundary of the experimental image in cylindrical coordinates, and the width and the length of the transformed image in Cartesian coordinates, respectively. In this problem, $y_{0}=r_{0}$, and $x_{0}$ can be arbitrarily chosen (here $x_{0}=r_{0}$ is used). As a result, the equation for the initial interface boundaries in Cartesian coordinates can be given as

$$
y=\frac{y_{p} \cos (\pi / N)}{\cos \left(n \pi / N-2 \pi x / x_{0}\right)}, \quad \frac{(n-1) x_{0}}{N} \leqslant x \leqslant \frac{n x_{0}}{N}, \quad n=1,2, \ldots, N,
$$

where $y_{p}=r_{p}$ corresponds to the distance from the apex to the geometric centre of the polygonal interfaces. Figure 17 presents an example of the transformation, in which the images of the initial octagon interface and two evolving interfaces at the instants before and after the arrival of the reflected shock in cylindrical coordinates are transformed into the images in Cartesian coordinates. In these images, $L_{a}$ and $L_{s}$ represent the distances from the focal point to the positions of the interface corresponding to the apex and the side centre, respectively. The measurements of $L_{a}$ and $L_{s}$ over each wavelength are approximatively taken using the distances from the leftmost and the rightmost points of the interface to the rightmost side of the image.

\subsection{Velocities of the interfaces}

Figure 18 plots the quantitative data of the interface features, including the normalized distances of movement (i.e. $L_{a} / L_{c}$ and $L_{s} / L_{c}$ ) and the normalized velocities (i.e. $W_{a} / W_{i 0}$ and $\left.W_{s} / W_{i 0}\right)$ of the interfaces corresponding to the apex and the side centre, respectively. The characteristic length, $L_{c}=r_{p}=20 \mathrm{~mm}$, and the characteristic time, $t_{c}=r_{p} / W_{i 0}=44.96 \mu \mathrm{s}$ are used, where $W_{i 0}=444.8 \mathrm{~m} \mathrm{~s}^{-1}$ denotes the velocity of the incident cylindrical shock wave when it arrives at the apex of the polygonal interface (i.e. the shock Mach number is 1.35). Note that the experimental data of $L_{a}$ 

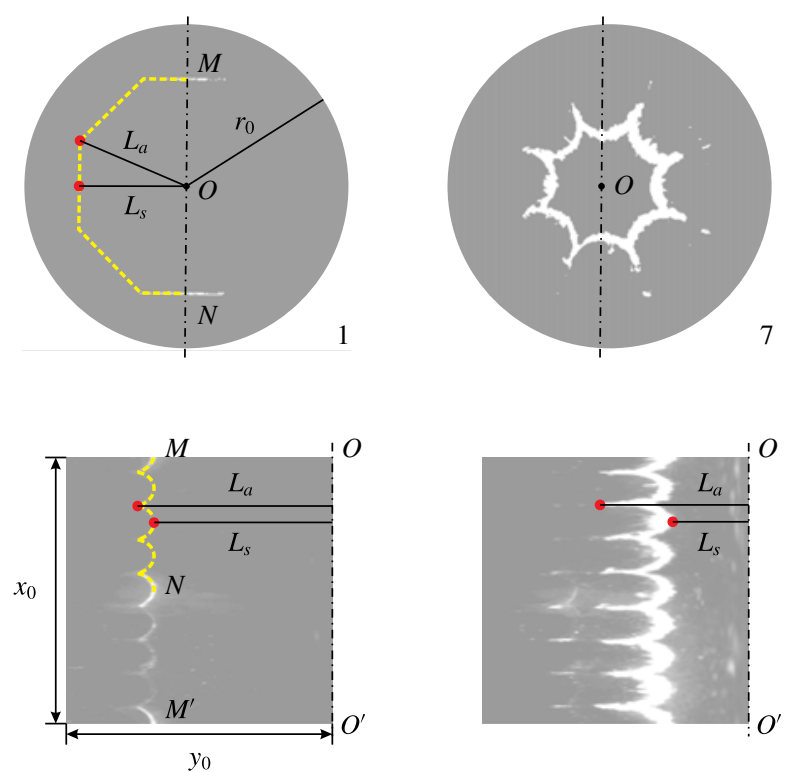
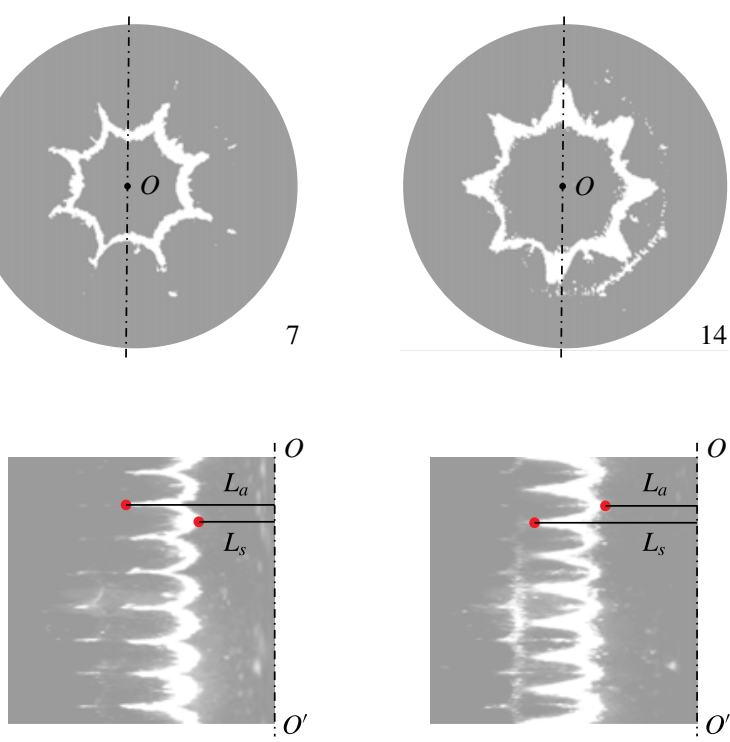

FIGURE 17. (Colour online) Images of the octagon interface showing the transformation from cylindrical to Cartesian coordinates. Experimental images correspond to frames 1, 7 and 14 in figure 9. $L_{c}$ and $L_{s}$ represent the distances measured from the focal point to the positions of the evolving interface corresponding to the apex and the side centre, respectively. The boundaries of the interface are highlighted by dashed yellow lines and the measurements of $L_{c}$ and $L_{s}$ in one of the wavelengths are indicated by the red dots.

and $L_{s}$ are measured for all wavelengths using averaged values in combination with error bars. The errors mainly come from the identification of interface boundaries and the difference between the evolving interfaces over different wavelengths. At the beginning, as shown in figure $18(a, b)$, both $L_{a}$ and $L_{s}$ decrease for all three interfaces. As the reflected shock passes across the evolving interface, $L_{s}$ begins growing because of the reversal of the interface, while $L_{a}$ first changes slightly with time in a short period and then decreases quickly as the first spikes emerging at the apexes of the interface are swallowed up by the bulk interface. Subsequently, $L_{a}$ decreases and $L_{s}$ increases gradually due to the development of the new bubbles and spikes. Although the trends of $L_{a}$ or $L_{s}$ changing with time for the three interfaces are similar, there are still some differences among them, which are heavily dependent on the initial conditions. On the one hand, when the incident cylindrical shock wave moves to the interface apexes, the shock strengths are nearly the same in all three cases (e.g. the shock Mach number is 1.35) and the difference among them mainly lies in their initial interface geometries (e.g. the shock-interface inclined angle $\phi$ is $22.5^{\circ}$ for the octagon, $45^{\circ}$ for the square, and $60^{\circ}$ for the equilateral triangle). On the other hand, when the incident shock wave moves to the interface side centres, the initial interface geometries are similar (e.g. the shock-interface angle is $0^{\circ}$ ), and the difference between them mainly comes from the shock strengths (e.g. the instantaneous incident cylindrical shock Mach number is 1.37 for the octagon, 1.45 for the square, and 1.55 for the equilateral triangle). The circulation initially deposited along the three polygonal interfaces shown in figure 16 are able to predict the interface movements qualitatively. 
(a)
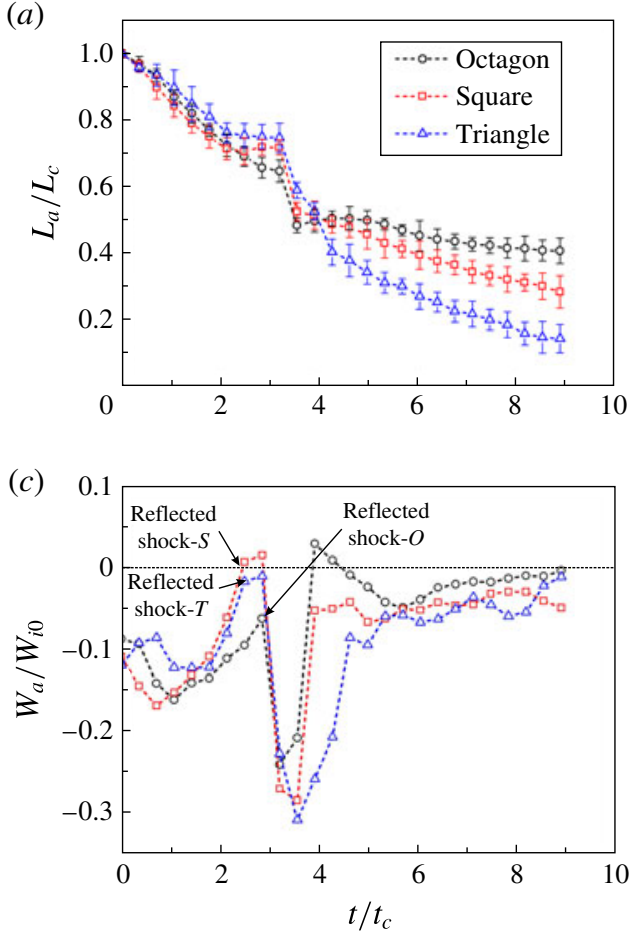

(b)

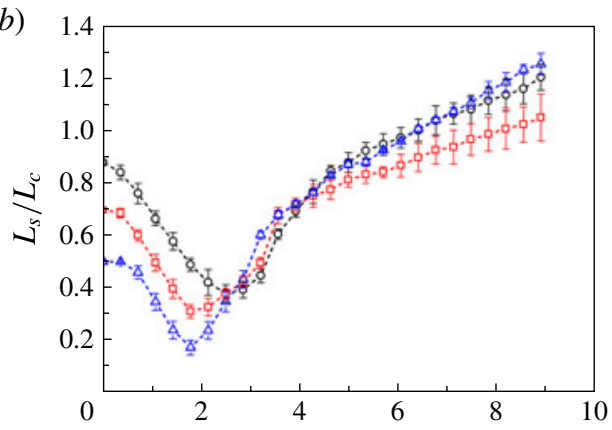

(d)

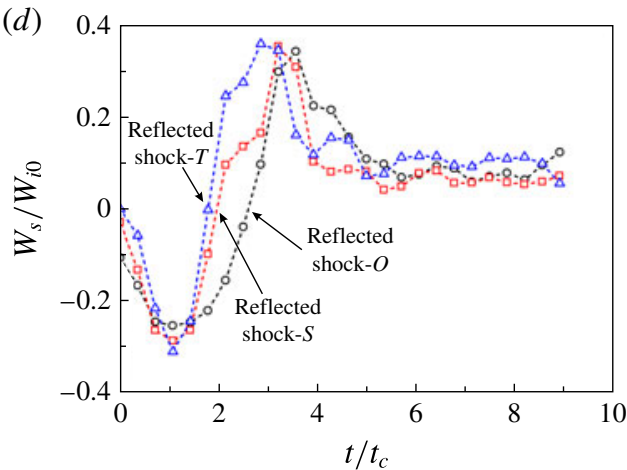

FIGURE 18. (Colour online) Quantitative data showing the normalized displacements of (a) the apex $L_{a} / L_{c}$ and $(b)$ the side centre $L_{s} / L_{c}$, and corresponding normalized velocities of $(c)$ the apex $W_{a} / W_{i 0}$ and $(d)$ the side centre $W_{s} / W_{i 0}$, of the polygonal gas cylinder, versus the dimensionless time $t / t_{c}$. In $(c)$ and $(d)$ the instants when the reflected shock impacts on the evolving interfaces corresponding to the apex and the side centre are estimated and marked by arrows and the velocity direction for the interface departing from the focal point is taken to be positive.

The corresponding velocities of the interfaces are approximatively calculated by obtaining the first derivatives of the averaged interface distances in the time domain, as shown in figure $18(c, d)$. The velocity direction for the interface departing from the focal point is taken as positive. The instants when the reflected shock impacts on the three types of evolving interface are also estimated. Unlike the planar shock cases (Wang et al. 2013; Zhai et al. 2014; Luo et al. 2015), the interface velocities change with time even in the very early stage because of the variable shock strength and the complex shock pattern during the interaction of the polygonal interface with the cylindrical shock. Also, for a planar air $/ \mathrm{SF}_{6}$ interface accelerated by an incident planar shock wave with the Mach number of 1.35 , the interface will attain a constant velocity of nearly $110 \mathrm{~m} \mathrm{~s}^{-1}$ according to shock dynamics theory. However, the dimensional interface velocities of the apex $W_{a}$ and the side centre $W_{s}$ after the passage of the incident cylindrical shock wave are much smaller than the theoretical predictions. As the presence of thin pins and fine chamfers in forming the initial polygonal interfaces has been evaluated to have little influence on the shock propagation and the interface evolution (Wang et al. 2013), the main reason for the difference would lie in the convergence effects. At the apexes of the interface, the velocities for the polygonal interfaces increase (except the triangular interface, whose velocity has a 
small reduction) at the beginning (e.g. $t / t_{c}<1$ ), and then decrease gradually to nearly zero (e.g. $1<t / t_{c}<2.5$ ). As the bubble-spike structures develop conversely (the original spikes diffuse and new bubbles arise in the vicinity of the apexes) because of the arrival of the reflected shock, there is a velocity jump in this period (e.g. $\left.3<t / t_{c}<4\right)$. After that, the velocities of the apexes become small with vibrations because of the shock reflections and refractions during the process (e.g. $t / t_{c}>5$ ). Before the arrival of the reflected shock (e.g. $1<t / t_{c}<2.5$ ), the octagonal interface moves more quickly at the apexes (i.e. spikes), while after the passage of the reflected shock (e.g. $t / t_{c}>5$ ), the apexes (i.e. bubbles) of the octagonal interface have a smaller averaged velocity. For the side centres of the interface, their velocities first increase from zero (e.g. $t / t_{c}<1$ ) because of the incident shock passage and then decrease to zero (e.g. $1<t / t_{c}<2$ ) because of the reflected shock arrival. The reflected shock arrives at the equilateral triangular, the square and the octagonal interfaces, sequentially. As the reversal of the interface appears after the passage of the reflected shock, the side centres of the interface are quickly accelerated to a high velocity. Then the interface moves away from the focal point with a decreasing velocity. In the late stage (e.g. $t / t_{c}>5$ ), the velocities of the side centre become nearly constant with small vibrations which can be ascribed to the consecutive reshocks in the converging cylindrical geometry.

The difference in the interface velocities corresponding to the apex and the side centre for the three cases, as shown in figure 18(c,d), further verifies the effect of initial conditions on the interface development. However, after the reflected shock impacts on the evolving interface, the flow field becomes complex, making the comparison among the three cases more difficult. As shown in figure 18, the variation of the square interface velocity differs from the other cases. The velocity of the square interface corresponding to the apex is the largest among the three cases in the early stage (e.g. $1<t / t_{c}<2.5$ ), and later becomes smaller than that for the equilateral triangular case and larger than that for the octagonal case (e.g. $t / t_{c}>5$ ). For the square case the velocity of the interface corresponding to the side centre becomes the smallest in the late stage (e.g. $t / t_{c}>5$ ). This phenomenon implies that the reflection, the refraction and the interaction of different shock patterns have an important influence on the interface evolution, because the complex shock patterns can change the vorticity generation and the circulation deposition in the complete process of interface evolution, which will be the next subject of our work.

\subsection{Growth rates of perturbations}

The variation with time of the interface features displays the characteristics of the RM instability induced by cylindrical shock waves. From the interface structural scales, the amplitude, $a$, of the evolving interface can be obtained, i.e.

$$
a(t)=\frac{1}{2}\left|L_{a}(t)-L_{s}(t)\right|,
$$

where the bars denote the absolute value. Then the growth rate $v(t)$ of the polygonal interface can be obtained by calculating the first derivative of the amplitude in the time domain, i.e. $v(t)=\dot{a}(t)$.

Figure 19 plots the normalized amplitudes $a / a_{i 0}$ and the normalized growth rates $v t_{c} / L_{c}$ (e.g. $v / W_{i 0}$ ) of the evolving interface varying with the dimensionless time $t / t_{c}$ for the three polygonal interfaces. At the very beginning a small fall of the amplitude arises because the incident shock compresses the apexes ahead of the sides of the interface. Before the arrival of the reflected shock, the value of $a / a_{i 0}$ increases because 

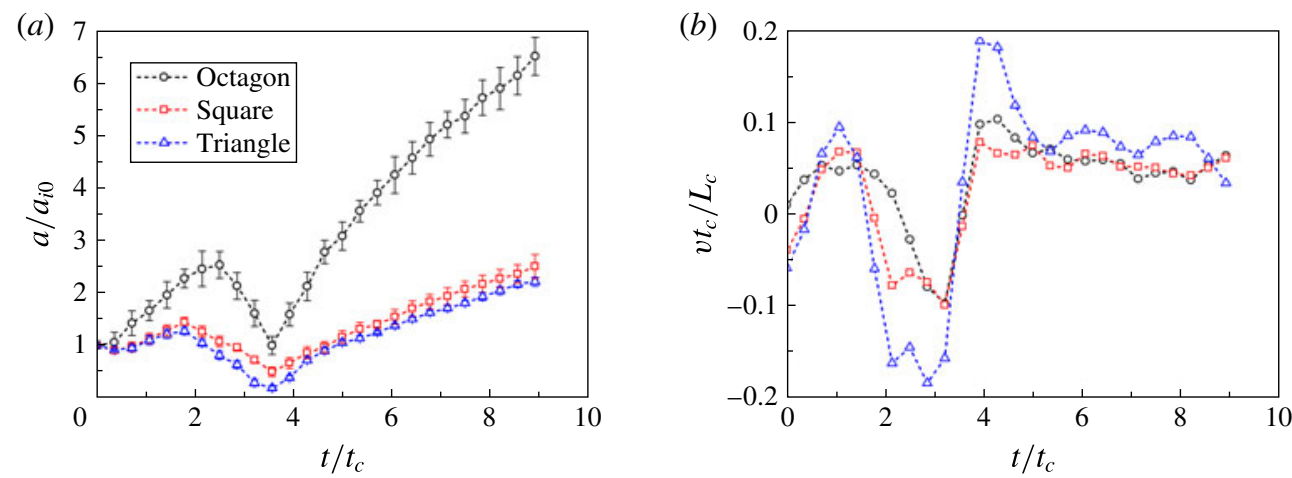

FIGURE 19. (Colour online) Quantitative data showing the normalized amplitudes $a / a_{i 0}$ (a) and corresponding normalized growth rates $v t_{c} / L_{c}(b)$ of the polygonal gas cylinder versus the dimensionless time $t / t_{c}$.

the side centre of the interface moves faster than the apex due to the convergence effects, as indicated in figure 18. Subsequently, the reversal of the evolving interface results in a reduction of the evolving interface amplitude. Later on, the value of $a / a_{i 0}$ increases continuously as the new spikes and bubbles are developing. During the process, the equilateral triangular interface always attains the smallest normalized amplitude, while the octagonal interface has the largest one among them at any instant. Moreover, the growth rates of the evolving polygonal interfaces seem distinct for these interfaces, as shown in figure $19(b)$. Strictly speaking, the time resolution in experiments is insufficient (e.g. the time interval between two consecutive frames is $16 \mu \mathrm{s})$, thus the growth rates of the evolving interface calculated by the first derivative of the amplitude in the time domain would have a small difference from the real situation. Nevertheless, the trends of the growth rates changing with time for these three cases, similar to those for the single-mode interface (Zhang \& Graham 1998), are reasonably acceptable. As the incident shock passes across the interface, the growth rate increases quickly. Then both the spikes and the bubbles move towards the focal point, resulting in the reduction of the interface growth rate. After the reflected shock passage, there is a reversal of the bubble-spike structures, and the growth rate becomes negative while its absolute value increases. As time proceeds, new spikes and bubbles are developing, resulting in the positive value of the growth rates. In the late stage, progressively weaker reshocks impact on the interface, and cause slight changes of the interface growth rates. As a whole, the variation of the interface growth rate comes from the movement of the bubble-spike structures, which is closely dependent on the shock systems interacting with the interface.

\section{Conclusion}

The interaction of cylindrical converging shock waves with a polygonal heavy gas cylinder is studied for the first time in a vertical annular diaphragmless shock tube. Three types of polygonal interfaces, with cross-sections of an octagon, a square and an equilateral triangle, are considered to evaluate the convergence effects and the baroclinic mechanism of the cylindrically converging RM instability with large initial deformation of the gas interfaces. First of all, the movements of the cylindrical shock and the reflected shock from the focal point are captured to validate the reliability of 
the shock tube facility. An analytical fitting self-similarity constant $\alpha=0.836 \pm 0.005$ is obtained by measuring the change in the shock positions with time. Then the complete evolution of these polygonal interfaces captured by a high-speed laser sheet imaging technique is presented. In the three cases, the incident cylindrical shock Mach numbers when the shock arrives at the initial interfaces are kept the same. Cylindrical bubble-spike structures are observed after the passage of the incident cylindrical shock. As the reflected shock from the focal point impacts on the evolving interface, phase inversion (reversal of the bubble-spike structure) appears. The interface deformation at the early stages is mainly attributed to the baroclinic vorticity generation. The total positive circulation deposited on the initial interface is calculated to be $3.73,3.25$ and $1.81 \mathrm{~m}^{2} \mathrm{~s}^{-1}$ for the equilateral triangular, the square and the octagonal interfaces, respectively. Quantitatively, the variations with time of the interface structures, including the interface displacements and the interface amplitudes, are measured, and the velocities of the interfaces and the growth rates of perturbations are also calculated. The results reported in this work are different from those in planar shock cases. In particular, the shock strength during imploding and exploding is different, which verifies the geometric convergence along the radial direction. Moreover, consecutive reshocks impact on the evolving interface, which suggests that compressibility plays an important role in the whole interaction process. Also, special behaviours of the movement of the interface structures are found. In a follow-up study, advanced flow visualization methods and numerical simulations will be employed to realize the flow morphologies and the shock patterns in the complete process quantitatively.

\section{Acknowledgements}

The authors appreciate the valuable comments of the anonymous referees. The authors also wish to acknowledge suggestions from Professor K. Takayama in Tohoku University on construction of the shock tube and thank $\mathrm{Mr}$ F. Lei, Ms H. He and Mr M. Wang for their help during the experiments. The work is supported by the National Natural Science Foundation of China (11272308 and 11302219) and the Fundamental Research Funds for Central Universities (WK2090050020).

\section{REFERENCES}

APAZIDIS, N. \& LeSser, M. B. 1996 On generation and convergence of polygonal-waves. J. Fluid Mech. 309, 301-319.

Apazidis, N., Lesser, M. B., Tillmark, N. \& Johansson, B. 2002 An experimental study of converging polygonal shock waves. Shock Waves 12, 39-58.

Arnett, W. D., Bahcall, J. N., Kirshner, R. P. \& Woosley, S. E. 1989 Supernova 1987a. Annu. Rev. Astron. Astrophys. 27, 629-700.

Balakumar, B. J., Orlicz, G. C., Ristorcelli, J. R., Balasubramanian, S., Prestridge, K. P. \& Tomkins, C. D. 2012 Turbulent mixing in a Richtmyer-Meshkov fluid layer after reshock: velocity and density statistics. J. Fluid Mech. 696, 67-93.

Balakumar, B. J., Orlicz, G. C., Tomkins, C. D. \& Prestridge, K. P. 2008 Simultaneous particle-image velocimetry-planar laser-induced fluorescence measurements of Richtmyer-Meshkov instability growth in a gas curtain with and without reshock. Phys. Fluids 20, 124103.

Balasubramanian, S., Orlicz, G. C., Prestridge, K. P. \& Balakumar, B. J. 2012 Experimental study of initial condition dependence on Richtmyer-Meshkov instability in the presence of reshock. Phys. Fluids 24, 034103. 
BARonets, P. 1994 Cylindrical shock waves generated by a spark discharge and their interaction with the shock waves from a pulsed induction discharge. Fluid Dyn. 29, 129-132.

Bates, K. R., NiKiforakis, N. \& HoldeR, D. 2007 Richtmyer-Meshkov instability induced by the interaction of a shock wave with a rectangular block of $\mathrm{SF}_{6}$. Phys. Fluids 19, 036101.

Brouillette, M. 2002 The Richtmyer-Meshkov instability. Annu. Rev. Fluid Mech. 31, 445-468.

Cohen, R. D. 1992 Shattering a liquid drop due to impact. Proc. R. Soc. Lond. A 435, 483-503.

Dimotakis, P. E. 2005 Turbulent mixing. Annu. Rev. Fluid Mech. 37, 329-356.

Dimotakis, P. E. \& SAmtaney, R. 2006 Planar shock cylindrical focusing by a perfect-gas lens. Phys. Fluids 18, 031705.

Guderley, G. 1942 Starke kugelige und zylindrische Verdichtungsstoesse in der Naehe des Kugelmittepunktes bzw. der Zylinderachse. Luftfahrtforschung 19, 302-312.

HaAs, J. F. \& Sturtevant, B. 1987 Interaction of weak shock waves with cylindrical and spherical gas inhomogeneities. J. Fluid Mech. 181, 41-76.

Hosseini, S. H. R., Onodera, O. \& TAKayama, K. 2000 Characteristics of an annular vertical diaphragmless shock tube. Shock Waves 10, 151-158.

Hosseini, S. H. R. \& TAKAYAma, K. 2005 Experimental study of Richtmyer-Meshkov instability induced by cylindrical shock waves. Phys. Fluids 17, 084101.

Hosseini, S. H. R. \& TAKAYAMA, K. 2010 Experimental study of toroidal shock wave focusing in a compact vertical annular diaphragmless shock tube. Shock Waves 20, 1-7.

Isenberg, C. 1992 The Science of Soap Films and Soap Bubbles. Dover.

JACOBS, J. W. \& SheEley, J. M. 1995 Experimental study of incompressible Richtmyer-Meshkov instability. Phys. Fluids 8, 405-415.

KJellander, M., Tillmark, N. \& Apazidis, N. 2011 Experimental determination of self-similarity constant for converging cylindrical shocks. Phys. Fluids 23, 116103.

Layes, G., Jourdan, G. \& Houas, L. 2003 Distortion of a spherical gaseous interface accelerated by a plane shock wave. Phys. Rev. Lett. 91, 174502.

Lindl, J. D., McCrory, R. L. \& CAmpbell, E. M. 1992 Progress toward ignition and burn propagation in inertial confinement fusion. Phys. Today 45, 32-40.

Lombardini, M., Pullin, D. I. \& Meiron, D. I. 2014a Turbulent mixing driven by spherical implosions. Part 1. Flow description and mixing-layer growth. J. Fluid Mech. 748, 85-112.

Lombardini, M., Pullin, D. I. \& Meiron, D. I. $2014 b$ Turbulent mixing driven by spherical implosions. Part 2. Turbulence statistics. J. Fluid Mech. 748, 113-142.

Long, C. C., Krivets, V. V., Greenough, J. A. \& Jacobs, J. W. 2009 Shock tube experiments and numerical simulation of the single-mode, three-dimensional Richtmyer-Meshkov instability. Phys. Fluids 21, 114104.

Luo, X., Si, T., YAng, J. \& Zhai, Z. 2014a A cylindrical converging shock tube for shock-interface studies. Rev. Sci. Instrum. 85, 015107.

Luo, X., WANG, M., Si, T. \& ZHAI, Z. 2015 On the interaction of a planar shock with an $\mathrm{SF}_{6}$ polygon. J. Fluid Mech. 773, 366-394.

Luo, X., WANG, X., Chen, M. \& ZhaI, Z. $2014 b$ Experimental study of shock interacting with well-controlled gas cylinder generated by soap film. J. Exp. Fluid Mech. 28, 7-13.

Luo, X., WANG, X.\& SI, T. 2013 The Richtmyer-Meshkov instability of a three-dimensional air/SF 6 interface with a minimum-surface feature. J. Fluid Mech. 722, R2.

Mariani, C., Vandenboomgaerde, M., Jourdan, G., Souffland, D. \& Houas, L. 2008 Richtmyer-Meshkov instability with stereolithographed interfaces. Phys. Rev. Lett. 100, 254503.

Matsuo, H. \& NAKAmuRA, Y. 1980 Experiments on cylindrically converging blast waves in atmospheric air. J. Appl. Phys. 51, 3126-3129.

Matsuo, H. \& Nakamura, Y. 1981 Cylindrically converging blast waves in air. J. Appl. Phys. 52, 4503-4507.

Meshrov, E. E. 1969 Instability of the interface of two gases accelerated by a shock wave. Fluid Dyn. 4, 101-104.

Orlicz, G. C., Balakumarand, B. J., Tomkins, C. D. \& Prestridge, K. P. 2009 A Mach number study of the Richtmyer-Meshkov instability in a varicose, heavy-gas curtain. Phys. Fluids 21, 064102. 
Perry, R. W. \& Kantrowitz, A. 1951 The production and stability of converging shock waves. J. Appl. Phys. 22, 878-886.

Ranjan, D., Anderson, M. H., OAKley, J. G. \& Bonazza, R. 2005 Experimental investigation of a strongly shocked gas bubble. Phys. Rev. Lett. 94, 184507.

Ranjan, D., OAKley, J. G. \& Bonazza, R. 2011 Shock-bubble interactions. Annu. Rev. Fluid Mech. 43, 117-140.

RichtmyeR, R. D. 1960 Taylor instability in shock acceleration of compressible fluids. Commun. Pure Appl. Maths 13, 297-319.

SAMTANEY, R. \& ZABUSKY, N. J. 1994 Circulation deposition on shock-accelerated planar and curved density-stratified interfaces: models and scaling laws. J. Fluid Mech. 269, 45-78.

SI, T., ZHAI, Z. \& LUO, X. $2014 a$ Experimental study of Richtmyer-Meshkov instability in a cylindrical converging shock tube. Laser Part. Beams 32, 343-351.

Si, T., ZhaI, Z., LuO, X. \& YANG, J. 2012 Experimental studies of reshocked spherical gas interfaces. Phys. Fluids 24, 054101.

Si, T., ZHAI, Z., LUO, X. \& YANG, J. $2014 b$ Experimental study on heavy-gas cylinder accelerated by cylindrical converging shock waves. Shock Waves 24, 3-9.

Takayama, K., Kleine, H. \& Gronig, H. 1987 An experimental investigation of the stability of converging cylindrical shock waves in air. Exp. Fluids 5, 315-322.

Tomkins, C. D., Kumar, S., Orlicz, G. C. \& Prestridge, K. P. 2008 An experimental investigation of mixing mechanisms in shock-accelerated flow. J. Fluid Mech. 611, 131-150.

Vandenboomgaerde, M. \& Aymard, C. 2011 Analytical theory for planar shock focusing through perfect gas lens and shock tube experiment designs. Phys. Fluids 23, 016101.

WANG, M., SI, T. \& LuO, X. 2013 Generation of polygonal gas interfaces by soap film for RichtmyerMeshkov instability study. Exp. Fluids 54, 1427-1435.

Watanabe, M., Onodera, O.\& TAKayama, K. 1995 Shock wave focusing in a vertical annular shock tube. Shock Waves 22, 99-104.

YAnG, J., KubotA, T. \& Zukoski, E. E. 1993 Applications of shock-induced mixing to supersonic combustion. AIAA J. 31, 854-862.

ZHAI, Z., LIU, C., QIN, F., YANG, J. \& LUO, X. 2010 Generation of cylindrical converging shock waves based on shock dynamics theory. Phys. Fluids 22, 041701.

Zhai, Z., Si, T., LuO, X.\& YANG, J. 2011 On the evolution of spherical gas interfaces accelerated by a planar shock wave. Phys. Fluids 23, 084104.

Zhai, Z., Si, T., Luo, X., Yang, J., LiU, C., TAN, D. \& Zou, L. 2012 Parametric study of cylindrical converging shock waves generated based on shock dynamics theory. Phys. Fluids 24, 026101.

Zhai, Z., WANG, M., SI, T. \& LUO, X. 2014 On the interaction of a planar shock with a light polygonal interface. J. Fluid Mech. 757, 800-816.

Zhang, Q. \& GRAhAM, M. J. 1998 A numerical study of Richtmyer-Meshkov instability driven by cylindrical shocks. Phys. Fluids 10, 974-992.

Zou, L., LiU, C., TAN, D., HuAnG, W. \& LUO, X. 2010 On interaction of shock wave with elliptic gas cylinder. J Vis. 13, 347-353. 